\title{
The Climate of Hd 189733b From Fourteen Transits and Eclipses Measured by Spitzer
}

\section{Citation}

Agol, Eric, Nicolas B. Cowan, Heather A. Knutson, Drake Deming, Jason H. Steffen, Gregory W. Henry, and David Charbonneau. 2010. "THE CLIMATE OF HD 189733b FROM FOURTEEN TRANSITS AND ECLIPSES MEASURED BYSPITZER.” The Astrophysical Journal 721 (2): 1861-77. https://doi.org/10.1088/0004-637x/721/2/1861.

\section{Permanent link}

http://nrs.harvard.edu/urn-3:HUL.InstRepos:41397413

\section{Terms of Use}

This article was downloaded from Harvard University's DASH repository, and is made available under the terms and conditions applicable to Other Posted Material, as set forth at http:// nrs.harvard.edu/urn-3:HUL.InstRepos:dash.current.terms-of-use\#LAA

\section{Share Your Story}

The Harvard community has made this article openly available. Please share how this access benefits you. Submit a story. 


\title{
THE CLIMATE OF HD 189733b FROM FOURTEEN TRANSITS AND ECLIPSES MEASURED BY SPITZER
}

\author{
Eric Agol ${ }^{1,2}$, Nicolas B. Cowan ${ }^{1}$, Heather A. Knutson ${ }^{3}$, Drake Deming ${ }^{4}$, Jason H. Steffen $^{5}$, Gregory W. Henry ${ }^{6}$, \\ AND DAVID CHARBONNEAU ${ }^{7}$ \\ ${ }^{1}$ Department of Astronomy, Box 351580, University of Washington, Seattle, WA 98195, USA \\ ${ }^{2}$ Kavli Institute for Theoretical Physics and Department of Physics, Kohn Hall, University of California, Santa Barbara, CA 93110, USA \\ ${ }^{3}$ Department of Astronomy, 601 Campbell Hall, University of California, Berkeley, CA 94720, USA \\ ${ }^{4}$ NASA's Goddard Space Flight Center, Planetary Systems Laboratory, Code 693, Greenbelt, MD 20771, USA \\ ${ }^{5}$ Fermilab Center for Particle Astrophysics, P.O. Box 500 MS 127, Batavia, IL 60510, USA \\ ${ }^{6}$ Center of Excellence in Information Systems, Tennessee State University, 3500 John A. Merritt Boulevard, Box 9501, Nashville, TN 37209, USA \\ ${ }^{7}$ Harvard-Smithsonian Center for Astrophysics, 60 Garden Street, Cambridge, MA 02138, USA \\ Received 2010 March 10; accepted 2010 July 25; published 2010 September 14
}

\begin{abstract}
We present observations of six transits and six eclipses of the transiting planet system HD 189733 taken with the Spitzer Space Telescope's Infrared Array Camera (IRAC) at $8 \mu \mathrm{m}$, as well as a re-analysis of previously published data. We use several novel techniques in our data analysis, the most important of which is a new correction for the detector "ramp" variation with a double-exponential function, which performs better and is a better physical model for this detector variation. Our main scientific findings are (1) an upper limit on the variability of the dayside planet flux of 2.7\% (68\% confidence); (2) the most precise set of transit times measured for a transiting planet, with an average accuracy of $3 \mathrm{~s}$; (3) a lack of transit-timing variations, excluding the presence of second planets in this system above $20 \%$ of the mass of Mars in low-order mean-motion resonance at 95\% confidence; (4) a confirmation of the planet's phase variation, finding the night side is $64 \%$ as bright as the day side, as well as an upper limit on the nightside variability of $17 \%$ (68\% confidence); (5) a better correction for stellar variability at $8 \mu \mathrm{m}$ causing the phase function to peak $3.5 \mathrm{hr}$ before secondary eclipse, confirming that the advection and radiation timescales are comparable at the $8 \mu \mathrm{m}$ photosphere; (6) variation in the depth of transit, which possibly implies variations in the surface brightness of the portion of the star occulted by the planet, posing a fundamental limit on non-simultaneous multi-wavelength transit absorption measurements of planet atmospheres; (7) a measurement of the infrared limb darkening of the star, which is in good agreement with stellar atmosphere models; (8) an offset in the times of secondary eclipse of $69 \mathrm{~s}$, which is mostly accounted for by a $31 \mathrm{~s}$ light-travel time delay and $33 \mathrm{~s}$ delay due to the shift of ingress and egress by the planet hot spot; this confirms that the phase variation is due to an offset hot spot on the planet; (9) a retraction of the claimed eccentricity of this system due to the offset of secondary eclipse, which is now just an upper limit; and (10) high-precision measurements of the parameters of this system. These results were enabled by the exquisite photometric precision of Spitzer IRAC; for repeat observations the scatter is less than $0.35 \mathrm{mmag}$ over the 590 day timescale of our observations after decorrelating with detector parameters.
\end{abstract}

Key words: planetary systems

Online-only material: color figures

\section{INTRODUCTION}

The planet system HD 189733 (Bouchy et al. 2005) is one of the best-studied transiting planet systems due to two factors: its close proximity to our solar system, making its star one of the brightest transit host stars, and the large size of the planet relative to the star, making the transits particularly deep. After the secondary eclipse was first detected for this planet by Deming et al. (2006), Knutson et al. (2007) made a precise measurement of the phase variation of the planet over slightly more than half of an orbital period using the $8 \mu \mathrm{m}$ Infrared Array Camera (IRAC; Fazio et al. 2004) on the Spitzer Space Telescope (Werner et al. 2004). In addition to yielding a longitudinal map of the planet (Cowan \& Agol 2008) which indicated an offset peak in brightness, attributed to advection of energy by a superrotating equatorial jet (Showman \& Guillot 2002; Cooper \& Showman 2005), this observation also yielded the most precise measurement of the depth of secondary eclipse, as well the most precise times of transit and secondary eclipse, for any extrasolar planet. This motivated us to propose additional observations of six transits and six eclipses of this system with the goals of looking for secondary eclipse variability (e.g., Rauscher et al. 2007), looking for transit-timing variations (TTVs) due to other planets in the system (Agol et al. 2005; Holman \& Murray 2005), improving the measurement of the atmospheric absorption (e.g., Tinetti et al. 2007), and improving the measured system parameters for better characterization of the planet, hoststar, and orbit properties (Winn et al. 2007; Torres et al. 2008; Pont et al. 2007).

The favorable properties of HD 189733 have allowed detections of planet absorption and emission features, yielding possible evidence for water, sodium, methane, carbon dioxide, and carbon monoxide, as well as Rayleigh scattering at blue wavelengths (Grillmair et al. 2007; Tinetti et al. 2007; Barnes et al. 2007; Redfield et al. 2008; Barman 2008; Swain et al. 2008, 2009; Charbonneau et al. 2008; Sing et al. 2009; Madhusudhan \& Seager 2009; Pont et al. 2008; Lecavelier Des Etangs et al. 2008).

Despite being an active star (Moutou et al. 2007; Henry \& Winn 2008) which affects radial-velocity (RV) measurements, the planet mass is measured precisely to be $M_{\mathrm{p}}=$ $1.13 \pm 0.03 M_{\text {Jupiter }}$, while the RV measurements constrain the eccentricity to be $e<0.008$ (Boisse et al. 2009). The planet radius is slightly larger than that of Jupiter (Bakos et al. 2006b; 
Baines et al. 2007; Winn et al. 2007). The orbit of the planet is aligned well with the spin axis of the star (Winn et al. 2006; Triaud et al. 2009).

The deluge of observational constraints on this system has inspired a wide range of theoretical modeling. In particular, the measured phase variation can be qualitatively explained by general circulation models, such as Showman et al. (2009b) and Rauscher \& Menou (2010), while two-hemisphere models have more difficulty explaining the shape and variation of the phase function (Burrows et al. 2008); see the recent review by Showman et al. (2009a) for a detailed discussion of these models.

After describing our observations (Section 2), we give an account of our preliminary data reduction (Section 3), describing our outlier rejection (Section 3.1) and choice of centroiding algorithm (Section 3.2). In Section 4, we discuss aperture photometry, background subtraction (Section 4.1), and then detail our correction for the detector ramp variation (Section 4.2), including a new double-exponential model for the ramp (Section 4.2.3) and its performance (Section 4.2.4). We complete the description of data reduction with a discussion of our choice of aperture size (Section 4.3), conversion to Barycentric Julian Date (BJD; Section 4.4), and error analysis (Section 4.5).

With the preliminary fit for the ramp, we then simultaneously fit to the stellar variability and planet variability, outside of eclipse or transit, and demonstrate the high precision of Spitzer IRAC (Section 5). We then fit the photometry with transit and eclipse models (Section 6), and show that the secondary eclipse depth offset can be explained by light-travel time and the offset hot spot (Section 6.5). We compute a new ephemeris from our data (Section 6.3), and use the times of transits to place limits on the presence of companion planets (Section 6.4). We show that the transit depth appears to vary, which we hypothesize is due to variations in the stellar surface brightness within the path of the planet (Section 6.6) and we show that the dayside planet flux measured from the secondary eclipses appears not to vary within the uncertainties (Section 6.7). We discuss these results and compare to models in the conclusions (Section 7).

A preliminary analysis of these data were presented in Agol et al. (2009); however, we have since made significant improvements in the analysis, in particular an improved ramp function, so the results presented here are more reliable. These data were also used by Carter \& Winn (2010) to place a constraint on the oblateness of HD 189733b, while for the purposes of this paper we assume the planet to be spherical.

\section{OBSERVATIONS}

We were awarded Spitzer Guest Observing time during Cycle 4 to observe six transits and six secondary eclipses of HD 189733 with IRAC Channel 4 (PI: E. Agol, program ID 40238). For each visit, we obtained 44,160 exposures of $0.4 \mathrm{~s}$ each over $5 \mathrm{hr}$ each. We also re-analyze the transit and eclipse from Knutson et al. (2007) for a total of seven eclipses and seven transits. We chose IRAC Channel $4(8 \mu \mathrm{m})$ as it has been demonstrated to be the most stable IRAC band (e.g., Cowan et al. 2007 and references therein). Due to the brightness of the host star we made the observations in sub-array mode; this mode allows shorter exposure times $(0.4 \mathrm{~s})$ and faster readout, but sacrifices the larger field of view $(32 \times 32$ pixels rather than $256 \times$ 256 pixels, where each pixel is $1^{\prime \prime} .2$ ). We turned off dithering which is required for high-precision photometry due to the arraydependent sensitivity and detector ramp. We carried out aperture photometry with a range of radii from 1 to 7 pixels in $1 / 2$ pixel increments.

\section{DATA REDUCTION}

We performed our data reductions starting with the Basic Calibrated Data (BCD) processed with version S16.1.0 of the Spitzer IRAC pipeline. These data are corrected for dark current, flat-field variations, and detector nonlinearity; they are also converted to units of flux in mega-Jansky per steradian (MJy $\left.\mathrm{sr}^{-1}\right)$. After downloading and organizing the data, we first converted the images to units of photon counts (i.e., electrons) by multiplying by the gain (fits header keyword GAIN = $3.8 e^{-} / \mathrm{DN}$ ) and exposure time (EXPTIME $=0.32 \mathrm{~s}$ ), and dividing by the flux conversion factor (FLUXCONV = $0.2021 \mathrm{MJy} \mathrm{sr}^{-1}$ per DN s${ }^{-1}$ ). For our $0.32 \mathrm{~s}$ exposure time, this amounts to multiplying each pixel by $6.01682 e^{-}$per MJy sr ${ }^{-1}$. The elapsed time per exposure is FRAMTIME $=0.4 \mathrm{~s}$ due to a $0.08 \mathrm{~s}$ readout. We did not apply corrections for variation in pixel area or corrections to the flat field for a stellar source; however, we did estimate the impact of these corrections, and found them to be negligible.

\subsection{Outlier Rejection}

After conversion to counts, we flagged and cleaned the images of outliers, such as cosmic rays. We cleaned the images at the pixel level by rejecting outliers in the time series for each pixel. This worked well since the telescope pointed at nearly the same location for the entirety of each of our observations, so the flux in each pixel stayed relatively constant making outliers easy to flag. The pixel flux is affected by pointing variations, discussed in Section 3.2, so we did the outlier rejection by taking the difference between the pixel time series and a five-exposure $(2 \mathrm{~s})$ running median of the time series. The duration of the median was chosen to be shorter than the shortest of the pointing excursions, one of which occurred in the middle of the transit of the phase-function observation with a 1 pixel pointing change over $4 \mathrm{~s}$.

For each pixel, the median-subtracted time series was sorted. Then, the standard deviation was computed from the $68.3 \%$ confidence limits on the median-subtracted time series. Finally, outliers were flagged in the median-subtracted time series that differed by more than $4 \sigma$ from zero. The flagged pixels were then replaced by the five-exposure median.

This procedure performed well in eliminating cosmic rays and rogue pixels. After it was carried out, the photometry showed no significant outliers and, as we show below, was very close to the photon-noise limit.

\subsection{Centroiding}

Spitzer is affected by pointing variations that cause the star to change position slightly on the detector; this requires accurate centroiding to perform precise photometry. There are four varieties of pointing variations we observe in the data: small amplitude, short-timescale "jitter" which appears by eye as a damped random walk; a periodic pointing fluctuation which occurs on a timescale of $\sim 1 \mathrm{hr}$ with an amplitude of about 0.1 pixel; gradual drifts over long timescales; and occasional short-timescale sharp excursions, as mentioned in Section 3.1.

In the initial stages of our data reduction, we found that the photometry was extremely sensitive to the pointing drifts. Since the $8 \mu \mathrm{m}$ camera is undersampled, variations in pointing at the $\sim 0.1$ pixel level can lead to $\sim 10 \%$ variations in the flux of 

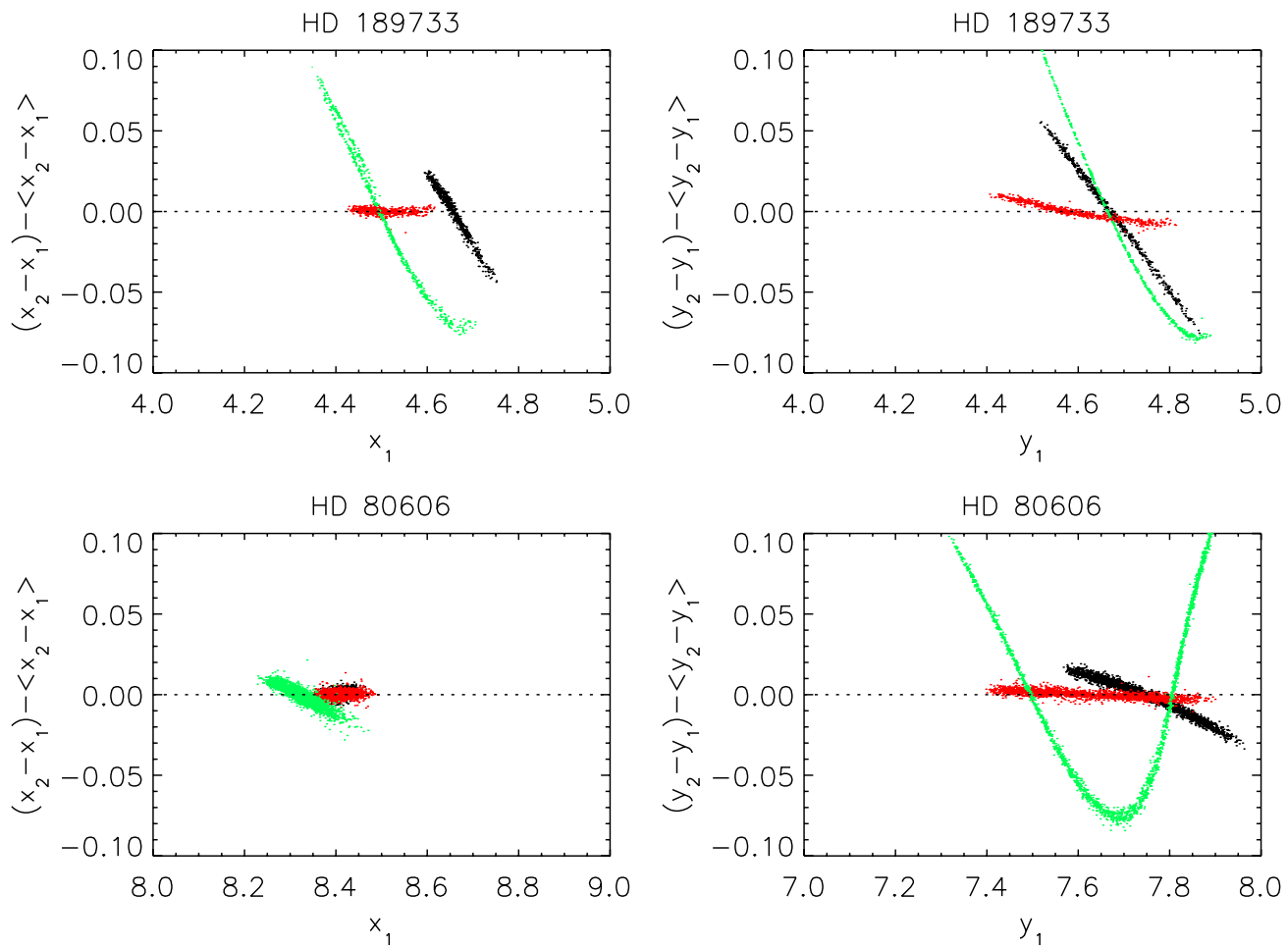

Figure 1. Comparison of the centroiding algorithms for HD 189733 vs. the M-dwarf companion (top panels) and HD 80606 vs. HD 80607 (bottom panels) in the $x$-direction (left panels) and the $y$-direction (right panels). The black dots are for the flux-weighted centroid, the red dots for the two-dimensional Gaussian centroid, and the green dots for the parabolic centroid. The HD 189733 data have been binned by 512 exposures (205 s), while the HD 80606 data have been binned by 4 exposures $(56 \mathrm{~s})$.

(A color version of this figure is available in the online journal.)

the central pixels. For large photometric apertures, this is not a problem since small changes in the centroid do not change the enclosed flux much. However, large apertures contain pixels with lower illumination which have a longer-lasting detector ramp (see Section 4.2), so a smaller aperture containing higher illumination pixels is more desirable since these pixels have a ramp that saturates more quickly. Thus, we realized that a very accurate centroiding algorithm would be necessary for using smaller apertures, so we set out to test a wide range of different centroiding algorithms to see which performed best. We tried several centroiding algorithms: flux-weighted centroiding (e.g., Knutson et al. 2007), parabolic fitting (e.g., Todorov et al. 2010), point response function (PRF) fitting (e.g., Laughlin et al. 2009), and two-dimensional Gaussian fitting (e.g., Désert et al. 2009). The simplest algorithms to implement are the first two since these involve no optimization; the last two involve iterative nonlinear optimization, but the PRF fitting turned out to be too slow and problematic to implement.

We first tested the centroiding algorithms by creating simulated jitter using the IRAC Channel 4 PRFs. From these tests, we found that the two-dimensional Gaussian fitting gave the least scatter in the derived centroid relative to the input centroid; we found that keeping the $x$ and $y$ standard deviations the same gave as good a fit to the centroid as allowing the two to vary independently. We next ran the algorithms on the two stars (the target star and the M-dwarf companion; Bakos et al. 2006a) in the phase-function data from Knutson et al. (2007) and on the two stars in the observations of HD 80606 (Laughlin et al. 2009). These tests were critical since these were long time series so the stars had time to drift a significant fraction of a pixel, and the data contain noise. We first computed the centroid of both stars in each image $\left(x_{1}, y_{1}\right.$ and $\left.x_{2}, y_{2}\right)$, then subtracted the $x$ and $y$ coordinates for each pair of stars $\left(x_{1}-x_{2}\right.$ and $\left.y_{1}-y_{2}\right)$, and finally binned the $x$ and $y$ differences until the standard deviation of the $x$ and $y$ differences reached a minimum. A perfect centroiding algorithm ought to have perfect tracking between the two stars, resulting in a standard deviation only due to the photon shot noise and finite spatial resolution of the instrument. Various choices can be made for each of these algorithms, such as what portion of the array to fit or whether to smooth the data first before centroiding, so we spent some time experimenting with these and other choices.

In short, we found that the two-dimensional Gaussian performed the best out of all the centroiding algorithms. The algorithm selects a $7 \times 7$ sub-array from the image centered on the brightest pixel of a star. It then fits a two-dimensional Gaussian to this sub-array, allowing the center (centroid), amplitude, and width to vary, four free parameters in all for each image. We used the mpcurvefit.pro routine which implements a nonlinear Levenberg-Marquardt algorithm to optimize these parameters (Markwardt 2009). For the HD 189733 phase-function observations, the scatter in the data was 0.0018 pixels in $x$ and 0.0051 pixels in $y$ when binned by 512 exposures $(205 \mathrm{~s})$, while for HD 80606 the scatter was 0.0015 and 0.0021 in $x$ and $y$ when binned by four exposures (56 s); further binning resulted in minimal decrease of the scatter. The second best centroiding algorithm was the flux-weighted algorithm which had standard deviations of 0.016 and 0.032 in $x$ and $y$ for HD 189733, and 0.0019 and 0.011 in $x$ and $y$ for HD 80606. Thus, the twodimensional Gaussian centroid performed better by a factor of $\sim 5$ than the flux-weighted centroid; not only that, but the scatter in the flux-weighted centroid is due to a systematic error, while the scatter in the two-dimensional Gaussian centroid is almost completely random. This can be seen in Figure 1 which shows 


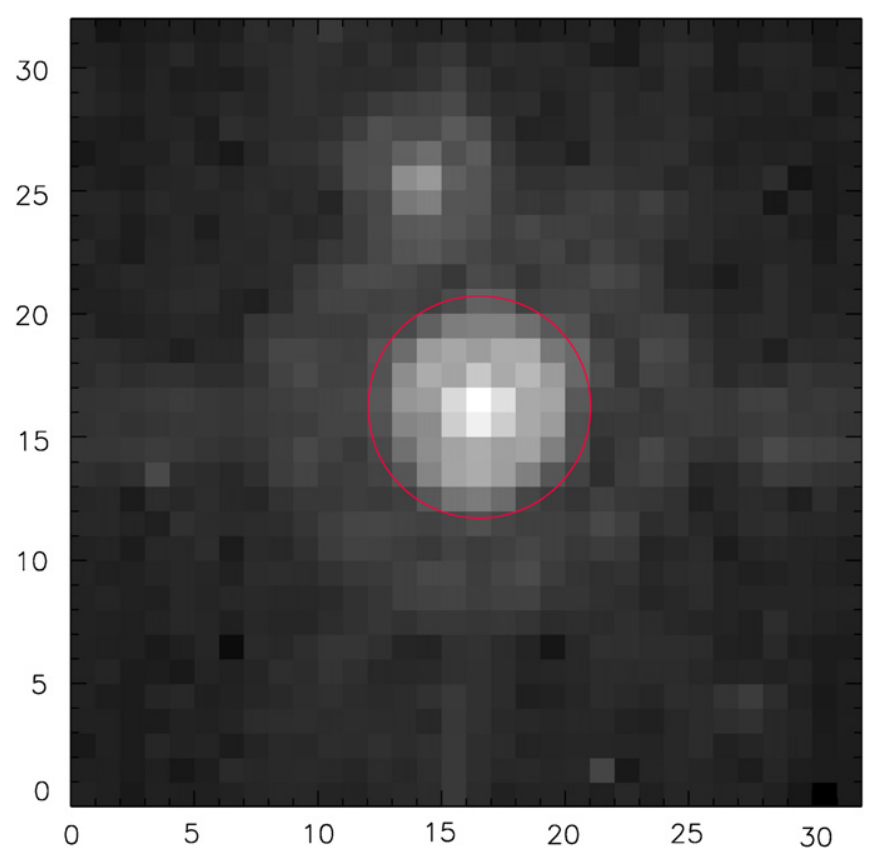

Figure 2. Logarithmic scaling of a medianed image from one of our observations; horizontal and vertical axes are pixels. White represents about 13,700 counts per pixel, while black is about 6 counts per pixel. The red circle is a 4.5 pixel radius aperture.

(A color version of this figure is available in the online journal.)

that the two-dimensional Gaussian centroid has weaker correlation of centroid difference of the two stars versus the centroid of one of the stars; on the other hand the other centroiding techniques show significant correlation between the offsets of the two stars and the pixel position of one of the stars, indicating a systematic error in the centroid determination.

Applying these centroiding algorithms to our 12 transits and eclipses and the transit and eclipse from Knutson et al. (2007), we find that the scatter in the difference in centroids of the two stars (adding in quadrature the $x$ and $y$ components) ranges from 0.0035 to 0.0042 pixels for the Gaussian centroid after binning by 128 exposures (51 s); this is a factor of 3-7 times smaller than the flux-weighted centroid, and also no systematic trend in $x$, and a weak systematic trend in $y$. Since the Gaussian centroids of the two stars track one another well and the difference in their positions is nearly uncorrelated with their position on the detector, we are confident that the Gaussian centroid is giving the correct absolute position of these stars. When the data are fit with a 3.5 pixel radius aperture (as described in more detail below), the two-dimensional Gaussian centroid yields a $\chi^{2}$ which is smaller for 9 of 14 transits/eclipses than the fluxweighted centroid, while the total $\chi^{2}$ is smaller by 130 (after discarding the first 55 minutes of data for each eclipse/transit which has the steepest portion of the ramp).

In conclusion, we recommend the two-dimensional Gaussian for Spitzer IRAC Channel 4 sub-array centroiding of bright targets as it appears to behave in a near-optimal manner. As we will show, this results in very small scatter in the resulting photometry.

\section{RAW PHOTOMETRY}

We carried out photometry on our data using aperture photometry with a circular aperture. The contribution of the pixels on the edges of the circle is calculated by multiplying the total flux in the pixel by the geometric fraction of the pixel that is covered by the circular aperture. This is done using the GSFC Astronomy Library IDL routine pixwt.pro. Figure 2 shows a logarithmically scaled median image from one of our sets of observations. As can be seen in the image, the sub-array is 32 pixels square, and a companion M-dwarf lies 9 pixels from the target star. We tried a range of apertures, discussed below in Section 4.3, but our final analysis uses a 4.5 pixel radius aperture which is shown as a red circle centered on the target star. Note that this aperture size contains the bulk of the target flux, and is near the minimum in flux just inside the first Airy ring; this makes our photometry less sensitive to variations in position. We have fit both stars with the measured PRF for IRAC Channel 4, and we find that the contribution of the M-dwarf within this aperture is less than $0.06 \%$ of the target star flux for all of our observations. The resulting light curves for our $12 \mathrm{ob}-$ servations plus the transit and eclipse from Knutson et al. (2007) are shown in Figure 3 for an aperture of 4.5 pixels radius. Note that for each transit/eclipse pair, the flux at eclipse is higher than the flux at transit; this indicates the planet is brighter on the day side than the night side.

\subsection{Background Subtraction}

To subtract the background, we used a similar procedure as that described in Knutson et al. (2007); namely, we fit a Gaussian to a histogram of the counts from a subset of pixels located in the corners of the image (excluding the M-dwarf companion, and excluding the top row). This background contributes about $1.9 \%-2.6 \%$ of the total flux in our 4.5 pixel radius aperture, which we subtract from the time series frame by frame. As discussed by Harrington et al. (2007) and in the IRAC Instrument Handbook, the flux and the background of the 1st-5th and 58th frame of every set of 64 exposures is systematically lower than the other exposures in a set. However, after we carried out background subtraction frame by frame, the offset in these exposures does not appear in our total time series. Consequently, we believe it is due to a bias offset that affects the entire frame uniformly, and thus is easily removed.

\subsection{Detector Ramp}

Spitzer was not envisioned as an instrument for carrying out high-precision photometry on bright targets; consequently it was designed without sub-millimagnitude exoplanet photometry in mind. An instrumental artifact that appears at the $\sim$ mmag level in photometry, but can be up to $10 \%$ for low-illumination pixels over $33 \mathrm{hr}$, is the so-called detector ramp (Deming 2009): a pixel which is illuminated uniformly in time shows a gradual increase in the detected flux (see Figure 3). This is an important effect to correct for in fitting photometric time series; unfortunately there has not been a full understanding of this effect for the Spitzer IRAC detector. Here, we derive a toy model which qualitatively matches the behavior of the ramp (Section 4.2.1). We show that prior functions used for ramp corrections in other analyses of IRAC data (e.g., Deming et al. 2006; Désert et al. 2009) do not have the correct functional form to describe the observed ramp (Section 4.2.2).

Understanding how to remove the detector ramp has evolved with time. Early work (Deming et al. 2006) ratioed the transit star to other sources, and modeled the baseline in the ratio as linear or quadratic in time. Fitting functions to the ramp directly have used either exponentials in time (Harrington et al. 2007) or polynominals in the log of time (Knutson et al. 2009; 


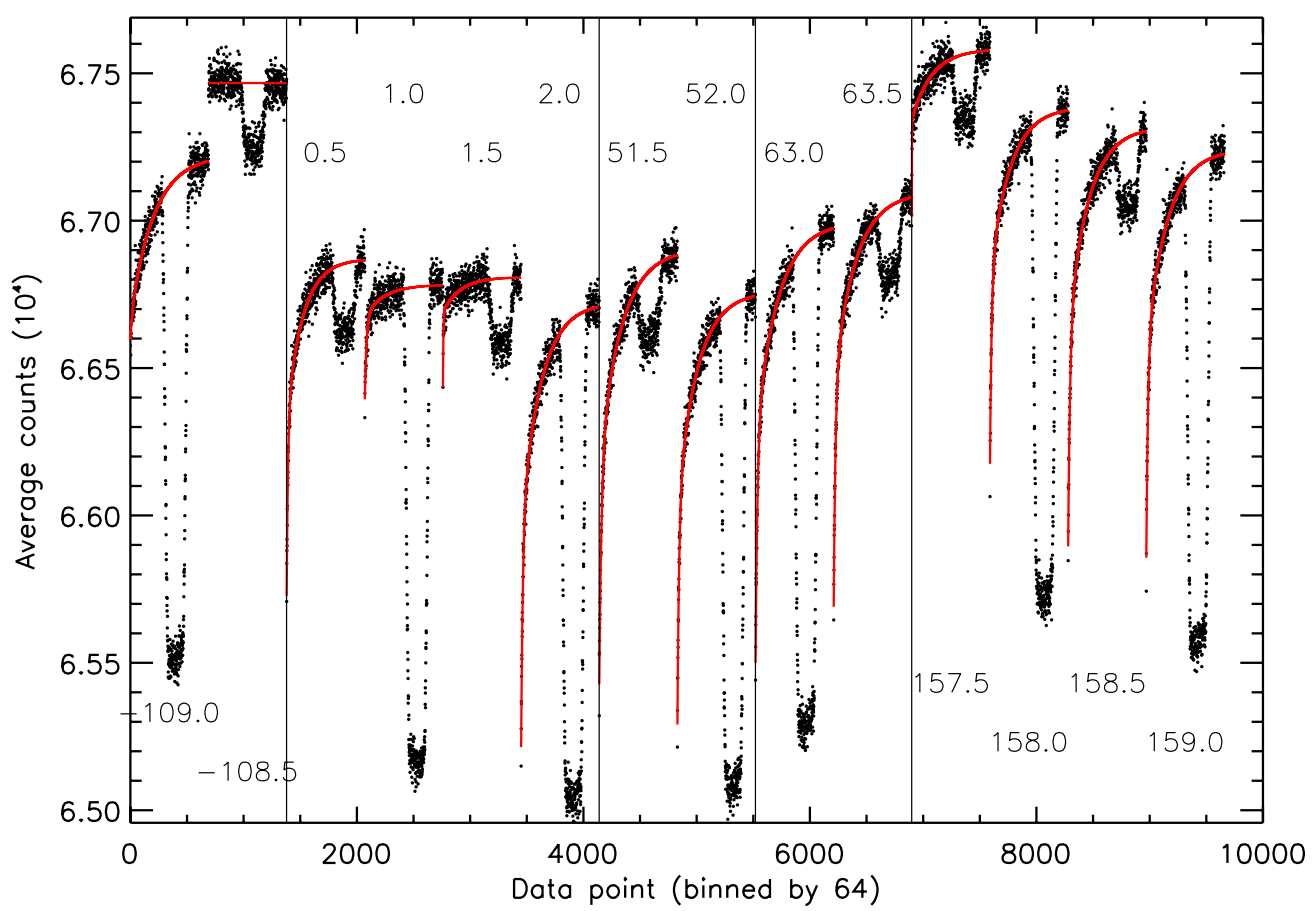

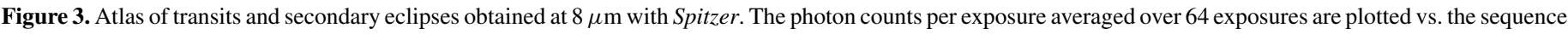

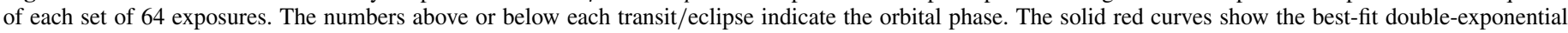
ramp model (which includes the phase variation of the planet for the secondary eclipses).

(A color version of this figure is available in the online journal.)

Désert et al. 2009). These approaches have been adequate at lower signal-to-noise ratios (S/Ns), but for the present high $\mathrm{S} / \mathrm{N}$ data we are motivated to find an improved functional form. We propose a new functional form, motivated by the toy model, which has the correct behavior and matches the observed ramps better (Section 4.2.3). We apply a range of tests to this ramp model, and show that it performs better than other ramp models (Section 4.2.4).

\subsubsection{Toy Model for the Detector Ramp}

The ramp effect is hypothesized to be due to trapping of electrons in detector defects ("charge trapping"). When a pixel is first illuminated, the charge traps are effectively empty, and some fraction of the electrons generated by the incident flux are retained by the traps instead of being read out by the array. As these charge traps fill, the effective gain of the detector goes up, until eventually the effect disappears. Thus bright, nonvariable sources should have a detected flux that asymptotes to a constant value. When a pixel is not illuminated (or illuminated at very low intensity), the trapped charge gradually releases with time, causing the charge traps to become empty; this leads to ghost images after exposure of bright sources. A consequence of this model is that higher illumination pixels fill their charge traps more quickly, thus showing a much shorter detector ramp timescale. Although it is not clear that this model is correct, its predicted behavior agrees qualitatively with the observed IRAC photometric properties: for a bright source, the central pixels have a short ramp which saturates quickly, while the pixels with lower illumination in the wings of the point-spread function (PSF) show a more gradual ramp. This behavior, though, is difficult to model quantitatively as the pixel illumination varies with time due to Spitzer pointing variations (see Section 3.2).

A simple toy model can be developed for charge trapping as follows. Let $\gamma_{\mathrm{m}}$ be the fraction of volume of a detector pixel filled with charge traps, $\gamma(t)$ be the fraction of volume of a pixel with empty charge traps at time $t$, and $\beta$ be the total well depth (electrons). A pixel is illuminated below saturation with an intensity causing $I(t)$ electrons to be released per second, while the measured intensity is $I^{\prime}(t)\left(e^{-} \mathrm{s}^{-1}\right)$. As the pixel is illuminated, the charge traps fill up at a rate proportional to the intensity times the fraction of volume of empty charge traps; however, there is also a timescale $\tau$ at which electrons in charge traps are released, causing $\gamma$ to increase. This gives

$$
\frac{d \gamma}{d t}=-\frac{I(t)}{\beta} \gamma-\frac{\gamma_{\mathrm{m}}-\gamma(t)}{\tau} .
$$

The measured intensity is then

$$
I^{\prime}(t)=(1-\gamma) I(t)+\beta \frac{\gamma_{\mathrm{m}}-\gamma}{\tau} .
$$

These equations have no closed-form solution for an arbitrary $I(t)$; however, we can solve for their behavior in certain limits. For instance, if the illuminating intensity is constant, $I(t)=I_{0}$, for times $t \geqslant t_{0}$, then

$$
\gamma(t)=\gamma_{\mathrm{m}}(1-I \tau / \beta)^{-1}+\left(\gamma\left(t_{0}\right)-\gamma_{\mathrm{m}}(1-I \tau / \beta)\right) e^{\left(\tau^{-1}-I / \beta\right)\left(t-t_{0}\right)} .
$$

As we are observing a bright star, we can simplify this equation by assuming that $\tau^{-1} \ll I_{0} / \beta$, but we are still below saturation and in the linear regime $\left(I_{0} t_{\exp } \lesssim 0.9 \beta\right.$, where $t_{\exp }$ is the exposure time). In this limit

$$
I^{\prime}(t) \approx I_{0}(1-\gamma)=I_{0}\left(1-\gamma\left(t_{0}\right) e^{-\frac{I_{0}}{\beta}\left(t-t_{0}\right)}\right) .
$$

This gives the expected ramp behavior: more strongly illuminated pixels have an apparent intensity, $I^{\prime}$, that asymptotes to a 
constant more quickly on a timescale $\beta / I_{0}$. At modest illumination, this becomes

$$
I^{\prime}(t)=I_{0}\left(1-\gamma_{0}+\gamma_{0} I_{0} \beta^{-1}\left(t-t_{0}\right)\right)
$$

a gradual linear ramp.

In the limit of zero illumination,

$$
\gamma(t)=\gamma_{\mathrm{m}}-\left(\gamma_{\mathrm{m}}-\gamma_{0}\right) e^{-\left(t-t_{0}\right) / \tau} .
$$

Thus, the apparent intensity is

$$
I^{\prime}(t)=\frac{\beta}{\tau}\left(\gamma_{\mathrm{m}}-\gamma_{0}\right) e^{-\left(t-t_{0}\right) / \tau}
$$

This leads to persistent or ghost images that decay exponentially with time after observation of a bright target when the illumination is so strong that $\gamma_{0} \ll \gamma_{\mathrm{m}}$.

\subsubsection{Prior Models for the Detector Ramp}

The correction for the detector ramp is typically applied after performing photometry on the target star rather than at the pixel level, with some exceptions (e.g., Knutson et al. 2007; Laughlin et al. 2009). There is a simple reason for this: at the pixel level it is very difficult to disentangle the ramp from pointing variations, while aperture photometry with a sufficiently wide aperture gets rid of most of the pointing variations and isolates the ramp behavior. Most ramp corrections have simply been functions that match the behavior of the ramp; two popular functions are $a_{0}+a_{1}\left(t-t_{0}\right)+a_{2} \log \left(t-t_{0}\right)$ (log linear) and $a_{0}+a_{1} \log \left(t-t_{0}\right)+a_{2}\left[\log \left(t-t_{0}\right)\right]^{2}$ (quadratic $\log$ ) which both seem to work well for IRAC Channel 4 data (e.g., Deming et al. 2006; Deming 2009). In particular, the logarithmic term matches the shape of the ramp well, which is steeper toward the beginning and shallower toward the end.

Given the toy model described in the prior section, this logarithmic behavior would appear to be largely coincidental. Aperture photometry combines pixels with a wide range of illuminations; those with high illumination, which is most of the flux, have a ramp that is steep and saturates quickly, while those with low illuminations in the wings of the PSF have a longer timescale ramp that saturates more slowly. Summing up these short- and long-timescale ramps gives a shape which is steeper in the beginning and more gradual as time passes, which is well modeled by a logarithmic function. The linear term or a squared logarithmic term gives enough extra degrees of freedom to the model to adjust the slope of the curve and gives a good fit to most ramp data. This model has the additional advantage that it is linear (except the initial time, $t_{0}$, in the logarithmic term), and thus is quick and easy to fit to the observed ramp.

However, the log-linear and quadratic log ramp models have a serious drawback: they do not have the correct behavior on long timescales. Both the log function and linear function increase without bound, while the detector ramp does appear to saturate at a constant value for the brightest pixels. Thus, with a data set with long duration, the log plus linear model or quadratic log ramp models should do a poor job in fitting the ramp shape. In addition, the log plus linear and quadratic log models do not describe what the final asymptotic flux value will be, and thus does not give a ramp correction, but only gives an empirical fit to how the flux is varying over the timescale of a given observation. These points are particularly important for small aperture photometry where most pixels have high illumination and thus saturate quickly. Consequently, we advocate not using ramps that are polynomials in time and/or log time.

\subsubsection{New Model for the Detector Ramp}

Motivated by the toy model in Section 4.2.1, we decided to try an exponential ramp function. As this model predicts, the time constant is a function of pixel illumination. However, due to the pointing variations, we were not successful in correcting for the ramp on the pixel level. Instead we tried a ramp correction function that is simply the sum of two exponential terms:

$$
F^{\prime} / F=a_{0}-a_{1} e^{-t / \tau_{1}}-a_{2} e^{-t / \tau_{2}},
$$

where $F^{\prime}$ is the flux affected by the ramp and $F$ is the flux corrected for the ramp. Although this does not have exactly the correct behavior for the sum of pixels with different illuminations (assuming the toy model is correct), it does have the correct asymptotic behavior, and qualitatively represents the correction from higher and lower illumination pixels.

Figure 3 shows the ramp function overplotted with our data for the 14 transits and eclipses.

\subsubsection{Performance of Double-exponential Ramp}

In addition to the qualitatively correct behavior of the doubleexponential ramp, we find that this ramp function leads to a smaller scatter in our derived eclipse depths for the seven eclipses, as well as less sensitivity to the various choices we make in our analysis. We held the planet-star radius ratio and impact parameter fixed at the transit values when analyzing the secondary eclipses. We ran initial fits for each ramp on photometry computed for a 3.5 pixel radius aperture with the first 55 minutes for each transit/eclipse discarded, and then determined how the eclipse depth changed as we varied individual analysis parameters. The scatter in the seven secondary eclipse depths for the double-exponential ramp model is smaller by $30 \%$ than for the log-linear ramp $(3.05 \%$ versus $3.94 \%$ ), and smaller by $20 \%$ compared to the quadratic log ramp $(3.05 \%$ versus $3.68 \%)$. An additional indication of the more robust behavior of this ramp function is that the mean depth only changes by $0.2 \%$ if we first fit the ramp to the outof-transit/eclipse data, and then fit the transit/eclipse to the ramp-corrected data versus a simultaneous fit to the transit/ eclipse and ramp. The log-linear and quadratic log ramps have a mean eclipse depth that changes by $0.5 \%$ and $1 \%$, respectively, between these two reduction techniques. Likewise, the doubleexponential ramp changes in eclipse depth by only $-1.1 \%$ if the first 55 minutes are discarded, while the log-linear and quadratic log change by $-1.2 \%$ and $3.7 \%$, respectively. The double-exponential ramp is also less sensitive to aperture size. For apertures between 3.5 and 5.0 pixels in radius, the individual eclipse depths vary by $1 \%$, while for the log-linear and quadratic $\log$ ramps, the variation is $1.5 \%$ and $2.4 \%$, respectively. Finally, the total $\chi^{2}$ for the double-exponential ramp model is slightly smaller by 21 than the log-linear ramp, and by 27 than the quadratic log ramp, which by the $F$-test for the additional 13 free parameters (for seven transits and six eclipses; the phasefunction eclipse has no ramp) favors the double-exponential ramp at $>99.999 \%$ confidence.

There are two drawbacks of the double-exponential ramp function: (1) it involves two nonlinear fit parameters, $\tau_{1}$ and $\tau_{2}$, which need to be optimized with a nonlinear minimizer and (2) in some cases when there is very little ramp (possibly due to high illumination prior to our observations), one or both of the $\tau$ values can diverge, or in some cases they can become degenerate. However, these drawbacks are straightforward to deal with by setting bounds in a nonlinear solver, and are 


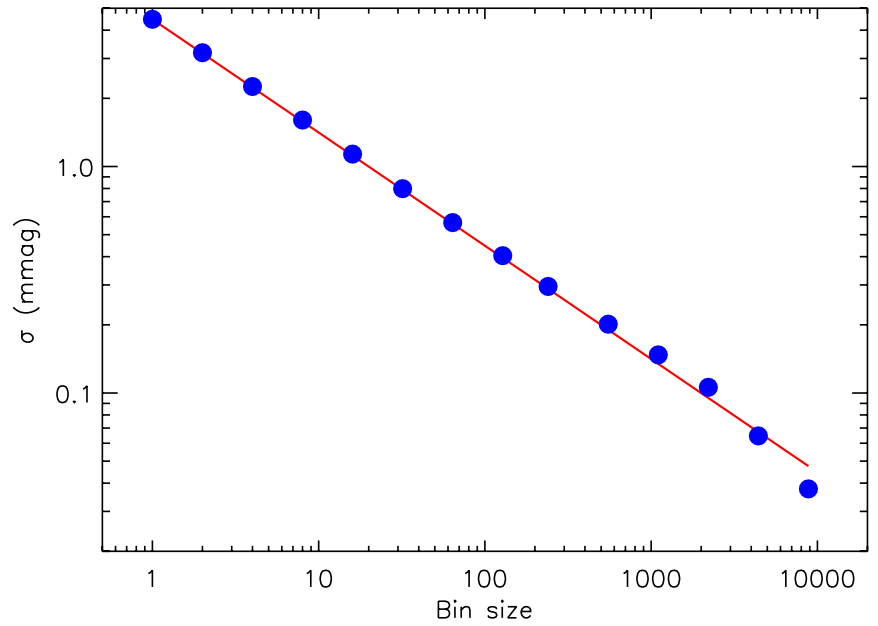

Figure 4. Scatter in the data (vertical axis) divided by the model binned by a number of bins (horizontal axis), averaged over the 14 transits and eclipses presented here. Red line is the extrapolation from the unbinned data by the inverse square root of the bin size.

(A color version of this figure is available in the online journal.)

outweighed by the improved fit to the observed ramp, the correct asymptotic behavior, the smaller scatter in our results, weaker dependence on aperture size, the weaker dependence on whether the ramp is first corrected or fit simultaneously, and less sensitivity to whether the steep portion of the ramp is discarded. Thus, we advocate using this ramp function for IRAC Channel 4 data.

\subsection{Aperture Size}

We carried out photometry with apertures ranging in radius from 1 pixel to 7 pixels. We fit each transit and eclipse separately, and then computed the standard deviation of the data divided by the best-fit model (this is essentially the residuals in magnitudes). We find that the residuals are minimized at 4.39 mmag for an aperture radius of 3.5 pixels; this is the same aperture chosen in Knutson et al. (2007). For aperture radii of 3.0 pixels, the scatter is $4.5 \mathrm{mmag}$, while for 4.0 pixels it is $4.40 \mathrm{mmag}$, and for 4.5 pixels is $4.47 \mathrm{mmag}$, indicating that there is a shallow dependence on scatter with aperture size.

More importantly, we wish to minimize the presence of red noise in the residuals of the data. Consequently, we looked at the scatter in the residuals binned over a range of bin sizes from one exposure to 1920 exposures as a function of aperture size. We then took the mean of the scatter of the binned data over all 14 transits and eclipses, and computed the product of this mean scatter divided by the unbinned scatter over all bin sizes. The minimum occurs for an aperture of 4.5 pixels; although this has a slightly larger residual scatter without binning, the binned residuals are smaller relative to the unbinned residuals than for the 3.5 pixel radius aperture case. We have also measured the power spectrum of the residuals, and we find that the 4.5 pixel radius aperture minimizes the long-period power. Thus, we feel that this aperture size represents an appropriate compromise between small scatter in the unbinned data (which varies weakly with aperture size) versus minimization of the red noise component.

Figure 4 shows the scatter in the binned residuals, averaged over the 14 transits, as a function of bin size. Even up to bin sizes of 8832 exposures ( $1 \mathrm{hr}$ bin), the scatter in the data does not deviate significantly from the inverse square root of the bin size; this indicates that the residuals are uncorrelated, and thus there is little (if any) red noise present. Remarkably the scatter in the $1 \mathrm{hr}$ bins reaches $30 \mu \mathrm{mag}$; however, this is after subtracting off the double-exponential ramp model.

For an aperture radius of 4.5 pixels, the median counts per exposure is 66,792 . If photon counting errors dominate, then the expected noise level is $3.87 \mathrm{mmag}$ per exposure. Including read noise $\left(4.5 e^{-}\right.$per pixel) and sky noise ( $\sim 30$ counts per pixel), the expected uncertainty is $3.99 \mathrm{mmag}$ (we did not use the BCD uncertainties since these overpredict the noise properties according to the Spitzer Observer's Manual). The standard deviation in the residuals is $4.47 \mathrm{mmag}$, which is only $15.5 \%$ greater than the photon counting error and $12.0 \%$ greater than the expected errors including read noise and sky noise. Thus, the noise properties after correcting for the detector ramp are very close to the expected photon noise. The 3.5 pixel radius aperture has a residual scatter which is only $11 \%$ above the photon noise; however, this aperture size appeared to have more significant red noise, so we opted for the larger 4.5 pixel radius aperture.

\subsection{Conversion to Barycentric Julian Date}

We use the JPL Horizons ephemeris for the Spitzer orbit to convert the satellite time (keyword DATE_OBS) to BJD in Coordinated Universal Time (UTC). ${ }^{8}$ This correction is important since heliocentric and BJD can differ by as much as a few seconds, and different time systems can vary by seconds depending on the number of leap seconds included, which is close to the timing precision we can achieve with these data (Eastman et al. 2010).

\subsection{Error Analysis}

We compute the errors on model parameters by calculating the residuals from each fit, shifting these by a random number of observations, and then adding the shifted residuals back to the best-fit model, then re-fitting; the so-called prayer-bead analysis (e.g., Agol \& Steffen 2007). For each transit and eclipse, we carried out 2000 iterations of the prayer-bead shifts. This has the advantage of preserving correlations in the noise of the data that might still be present. For instance, if the ramp model is incorrect, there may be systematic deviation due to using the wrong ramp model, and these deviations are preserved within the residuals. This approach has some disadvantages; for instance, if the noise behaves differently within eclipse than outside eclipse, this might exaggerate the noise outside eclipse. Another disadvantage of this technique is that the number of independent trials is limited by the size of the data set since point order has to be preserved; consequently, we also randomly chose to reverse the residuals or change their sign to give more independent noise realizations. There is also the possibility that the effects of correlated noise may be removed in the fit. Even with these disadvantages we expect that this technique gives a fairly conservative estimate for the uncertainties on model parameters.

\section{FIT FOR STELLAR AND PLANET VARIABILITY}

Stellar variability can affect our fits to the transits and eclipses, as well as our estimate of the planet variability. We follow the approaches in Knutson et al. (2009) and Sing et al. (2009) to

\footnotetext{
8 There is an additional $+65.184 \mathrm{~s}$ offset to convert to Barycentric Dynamical Time for our data.
} 
derive a new estimate of the relation between the optical and $8 \mu \mathrm{m}$ flux variability of the star by comparing our data set with that of Henry \& Winn (2008), plus additional unpublished data. The contemporaneous optical monitoring data were taken with the T10 $0.8 \mathrm{~m}$ automated photometric telescope at Fairborn Observatory, which has a median time sampling of 1 day, but gaps of up to 2 weeks. The optical time series consists of the mean of Stromgren $b$ and $y$ magnitudes, subtracted from a nearby comparison star, HD 189410, giving $f_{\mathrm{opt}}=\Delta[(b+y) / 2]$ as a function of time, as described in more detail in Winn et al. (2007). Data outliers are removed (usually taken in poor conditions), resulting in a total of 700 observations over five seasons.

Using the measured stellar rotation period of $P_{*}=11.953 \pm$ 0.009 days from Henry \& Winn (2008), we fit a sinusoidal function to data within each season to interpolate the measured optical fluxes to the times of our Spitzer observations. For all but one of our IRAC observations, there was at least one optical observation taken within one day, and all within two days. We then computed the total unocculted $8 \mu \mathrm{m}$ flux, $f_{\text {ir }}$, at the mid-transit and mid-eclipse times of our 14 observations, after correcting for the best-fit double-exponential ramp, to look for a correlation between the $8 \mu \mathrm{m}$ and optical fluxes. The initial data seemed to show little correlation between the infrared and optical fluxes, so we carried out a regression of the infrared fluxes against five variables: (1) the optical flux, $f_{\text {opt }}$, which is entirely due to the star; (2) the phase $\Phi$ which determines whether the source is transiting or eclipsing (i.e., whether we are seeing the day or night side of the planet), $\Phi=0$ during transit and $\Phi=1$ during secondary eclipse; (3) the average centroid position, $x_{\mathrm{c}}$, on the detector for each of our observations (the $y$ position varied little between observations); (4) the average infrared background flux scaled to our aperture, $f_{\text {bkd }}$ (this was already subtracted in earlier analysis of the data, but we nevertheless include it in the regression); and (5) the amplitude of the first exponential ramp, $a_{1}$. The last three terms are included to take into account the possibility of flat-field errors, imperfect background subtraction, and the imperfect performance of our ramp function.

We find the best-fit relation

$$
\begin{aligned}
\frac{f_{\text {ir }}}{\left\langle f_{\text {ir }}\right\rangle}-1= & (0.197 \pm 0.022) \frac{f_{\text {opt }}-\left\langle f_{\text {opt }}\right\rangle}{\left\langle f_{\text {opt }}\right\rangle}+(1.044 \pm 0.026) \frac{f_{\text {bkd }}}{\left\langle f_{\text {ir }}\right\rangle} \\
& -(6.59 \pm 0.84) \times 10^{-4} x_{\mathrm{c}}+(1.19 \pm 0.16) \times 10^{-3} \Phi \\
& +(1.55 \pm 0.48) \times 10^{-6} a_{1}-(12.7 \pm 1.2) \times 10^{-3},
\end{aligned}
$$

where $\left\langle f_{\text {ir }}\right\rangle$ is the ramp-corrected flux averaged over all 14 observations, while $\left\langle f_{\text {opt }}\right\rangle$ is the average over the optical flux at the times of the 14 observations. The left-hand side of this relation is plotted versus the right-hand side in Figure 5; the scatter about this relation is $0.35 \mathrm{mmag}$. We have computed the uncertainties on the regression coefficients by Monte Carlo simulation.

The standard deviation of the residuals of our sinusoidal fits to the optical data is $2.5 \mathrm{mmag}$ (after exclusion of a few outliers), which is 1.8 times the optical flux uncertainty ( 1.4 mmag; Henry $\&$ Winn 2008). Using the sinusoidal fits, the uncertainty on the optical flux we predict at the mid-points of our observed transits and eclipses ranges from 0.6 to $1.4 \mathrm{mmag}$ after inflating the optical errors by a factor of 1.8. Since infrared stellar fluctuations are $20 \%$ of the optical, this predicts a scatter of $0.1-0.3 \mathrm{mmag}$ in the infrared, which is consistent with the measured scatter.

We have computed the expected spectral change at $8 \mu \mathrm{m}$ compared to $(b+y) / 2$ for a star spot model in which the star spots

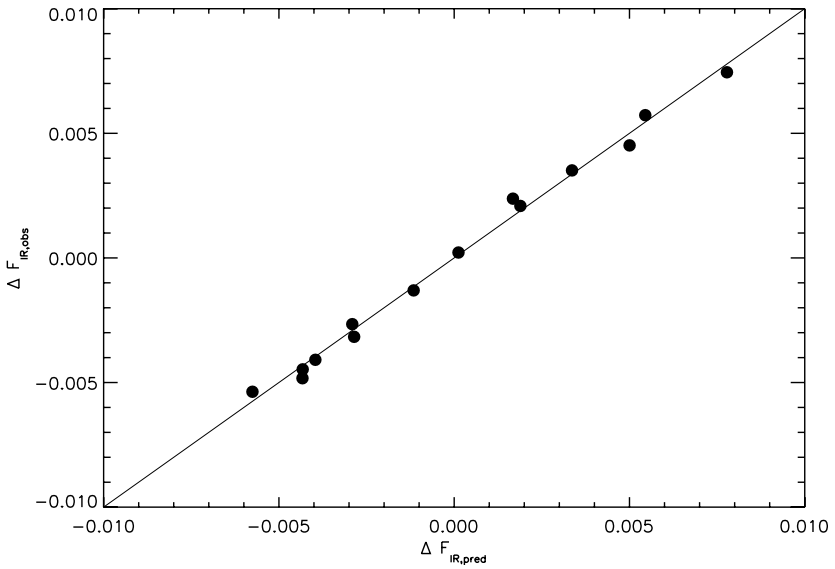

Figure 5. Left-hand side of Equation (9) vs. the right-hand side. Solid line is equality; the vertical scatter in the residuals of this relation is $0.35 \mathrm{mmag}$.

are modeled as Kurucz stellar atmospheres (Kurucz 1992) with $4000-4500 \mathrm{~K}$ (which is the estimated temperature from occulted star spots measured with Hubble Space Telescope (HST) by Pont et al. 2008), while the bulk of the star is $5000 \mathrm{~K}$, following the procedure described in Knutson et al. (2009). We find the expected change at $8 \mu \mathrm{m}$ is $21 \%-23 \%$ of the change in the optical, very close to our measured value, the first coefficient in Equation (9).

There are several important implications of this relation: (1) as the scatter in this relation is only $0.35 \mathrm{mmag}$, this indicates that photometry with Spitzer is reproducible to $0.35 \mathrm{mmag}$ over a 590 day period; (2) this scatter limits our uncertainty on the measurement of the nightside planet flux (during transit), so we can claim that the nightside variability is less than $0.35 \mathrm{mmag}$, which is about $17 \%$ of the planet's nightside flux or $10 \%$ of the planet's dayside flux (which we fit for in Section 6.7); (3) the transits are $1.19 \pm 0.16$ mmag fainter than the eclipses-after accounting for stellar variability — due to the cooler night side of the planet than the day side, confirming the phase variation detected in Knutson et al. (2007); and (4) the infrared flux variations are about $20 \%$ of the optical variations.

The derived infrared/optical correlation is nearly twice the value derived in Knutson et al. (2009), which used a smaller subset of data to carry out the correlation and thus was unable to regress against these other factors. Our estimate of the expected flux variations from Kurucz stellar atmospheres indicates that our derived value is likely correct. However, Knutson et al. (2009) derived a larger change in the stellar flux-by interpolating the observed $y$-band light curve-than we obtained by sinusoidal fitting of the $(b+y) / 2$ light curve over the period of duration of the phase-function observation, so the resulting estimates of $8 \mu \mathrm{m}$ stellar variation for the Knutson et al. (2007) observation are nearly the same: a 0.6 mmag increase in stellar flux between transit and eclipse.

\section{ECLIPSE AND TRANSIT MODELS}

We fit a model of a straight-lined trajectory of the planet over the disk of the star. To compute the transits and eclipses, we used the analytic formulae from Mandel \& Agol (2002), treating the planet as a uniform disk (no limb darkening), and the star as a disk with a linear limb-darkening law.

For each transit, the model has six physical parameters and four ramp parameters: the stellar flux $F_{*}$, the sky velocity $v$ (units of stellar radius per day), the impact parameter $b$ (units of stellar radius), the planet-star radius ratio $p=R_{\mathrm{p}} / R_{*}$ 

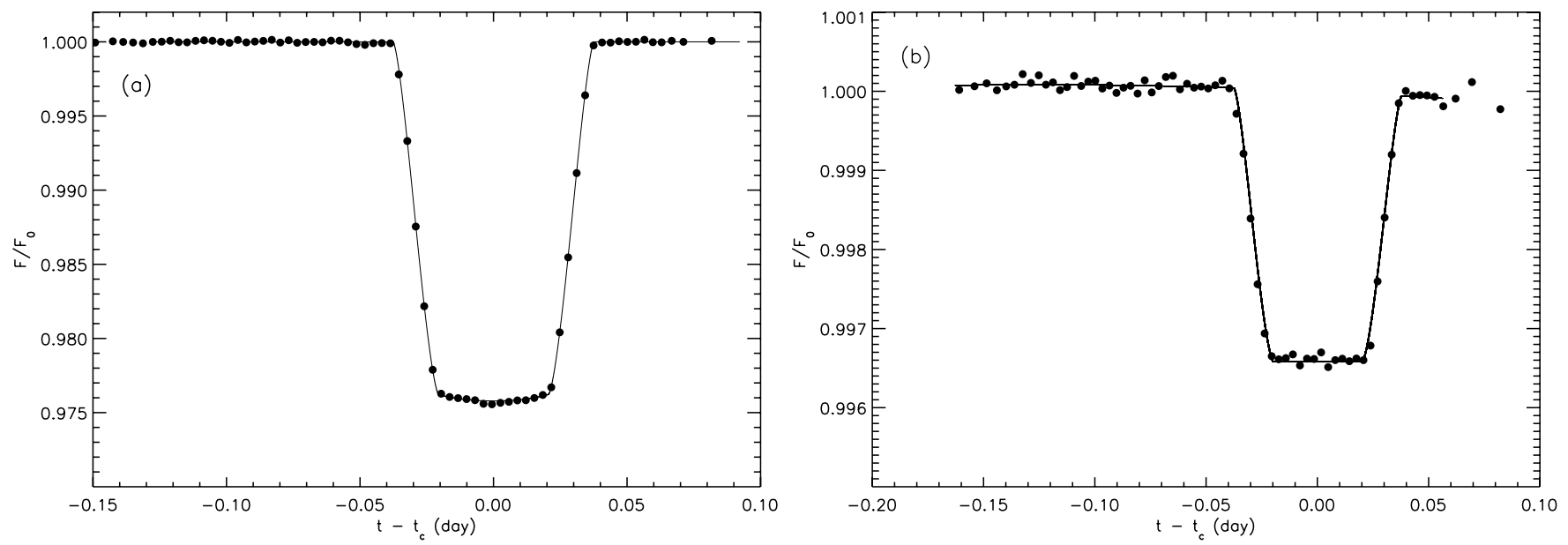

Figure 6. (a) Average light curve for the seven transits and (b) seven eclipses with best-fit models (solid lines). The data have been binned by 8960 data points to a total of 69 data points for each.
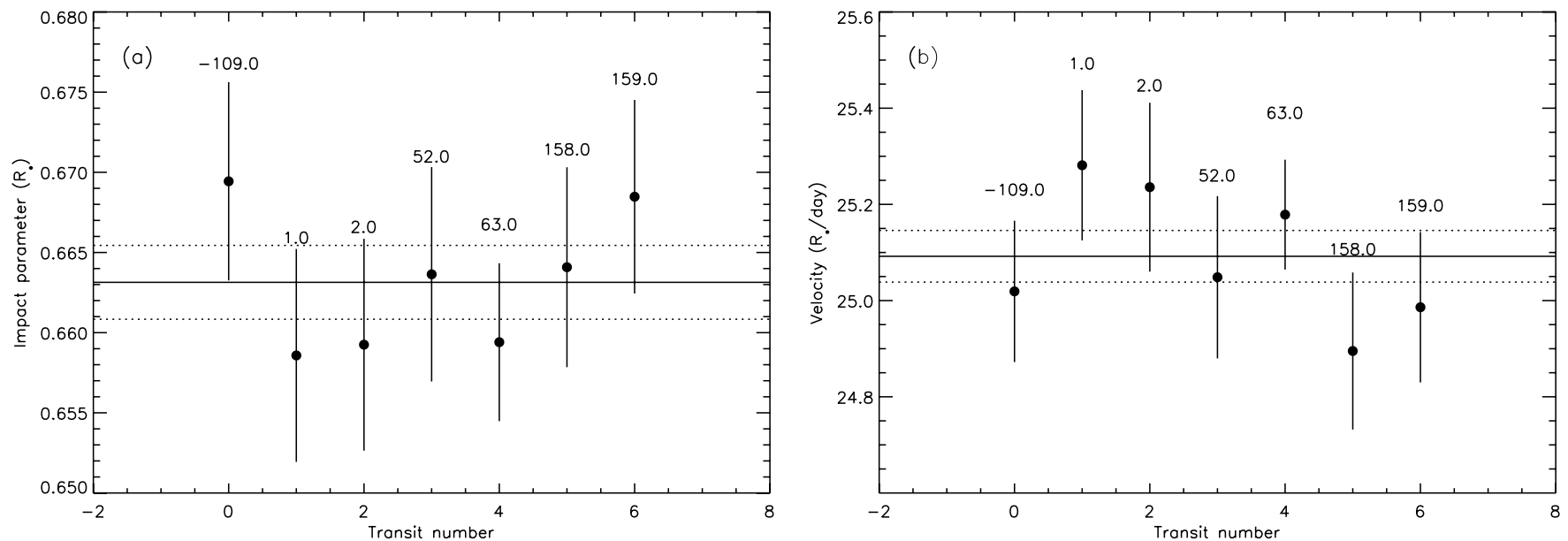

Figure 7. (a) Impact parameter and (b) transit velocity, vs. transit number with estimated error bars. The horizontal solid line is the average of the seven measurements, while the dotted lines are the uncertainties on the average values. The numbers above each data point show the number of periods before or after the zero point of our measured ephemeris.

(dimensionless), the time of central transit $t_{\mathrm{c}}$ (in BJD), the linear limb-darkening parameter of the star $u_{1}$ (dimensionless), and the double-exponential ramp parameters $a_{1}, \tau_{1}, a_{2}$, and $\tau_{2}$ (Equation (8)). Note that we neglect the contribution of the planet's flux during transit; this is because we find this is completely degenerate with the transit depth, and thus leads to problems in fitting (Kipping \& Tinetti 2009). We initially neglected phase variation of the planet and variation of the flux of the star during transits as the ramp affects all of the transit data sets and thus a short-timescale $(5 \mathrm{hr})$ planet or stellar variation cannot be disentangled from the ramp for a single observation.

For the secondary eclipses, we assumed that the planet phase function followed the same shape as that of Knutson et al. (2007), which we held fixed in our fits to each secondary eclipse, but we allowed the total planet flux to vary for each eclipse. For each secondary eclipse, we held fixed the planet/star radius ratio $p$, the impact parameter $b$, and the velocity $v$, at the best-fit values from the transit observations; these parameters are poorly constrained by the secondary eclipses, and holding them fixed has no impact on the fitting. Thus, for each secondary eclipse we have three physical parameters that are varied: the stellar flux $F_{*}$, the planet flux $F_{\mathrm{p}}$, the central time of eclipse $t_{\mathrm{c}}$, as well as the four ramp parameters.

\subsection{Results from Fits to Individual Transits/Eclipses}

We allowed the model parameters to vary independently for each transit/eclipse. These fits were necessary since a simultaneous fit to the entire data set is computationally intensive due to the large number of data points; we avoided pre-binning the data to preserve as much information as possible about the noise in the final results. Figure 6 shows the average of all seven transits and all seven eclipses, corrected for the detector ramp and folded to the same orbital phase. We have binned the data by 8960 exposures to 69 data points for clarity.

\subsection{Transit Impact Parameter and Sky Velocity}

For the transits, we found that the sky velocity, $v$, and impact parameter, $b$, have no evidence for variation. Figure 7 shows each of these parameters plotted versus the transit number. The sky velocity has an average fractional uncertainty of $0.62 \%$; the scatter in the measured values is $0.57 \%$, and thus is consistent with being constant. Combining our data together, we find the average sky velocity is $25.125 \pm 0.064 R_{*}$ day $^{-1}$, while a limit on the variation of the sky velocity is $d v / d t=(-5.5 \pm 6.6) \times$ $10^{-4} R_{*}$ day $^{-2}$. The impact parameter has an average measured value of $0.6631 \pm 0.0023 R_{*}$, with an average fractional uncertainty for each observation of $0.93 \%$ and a fractional 

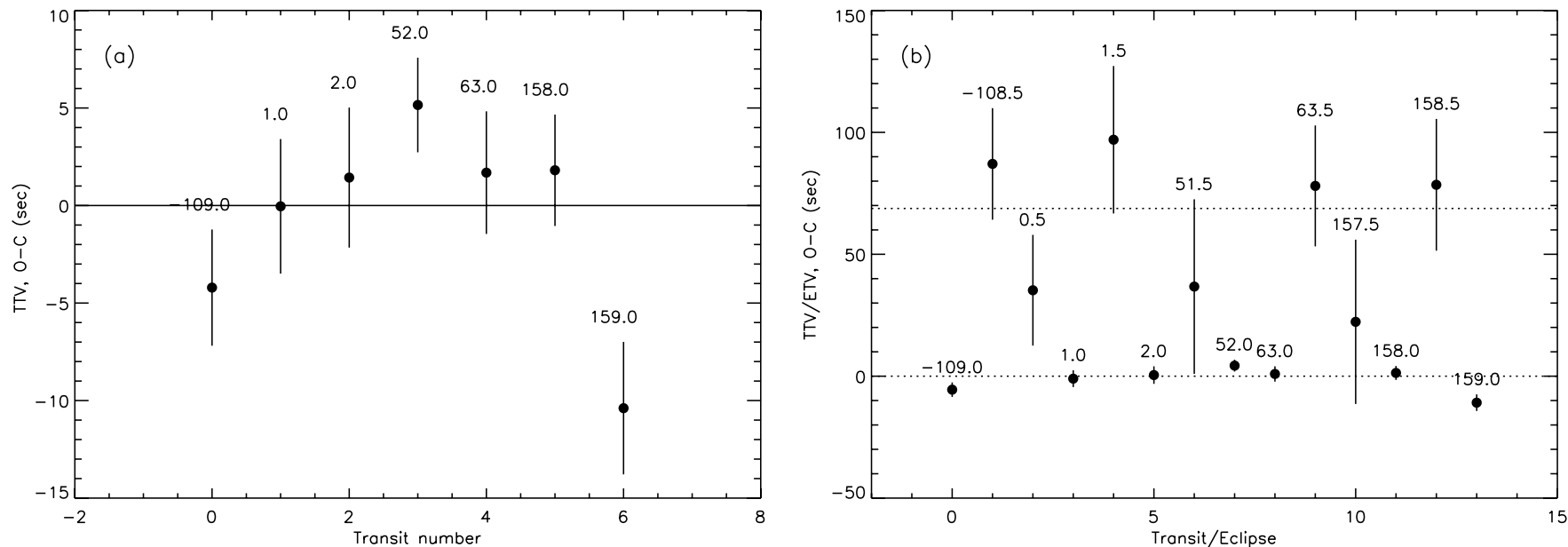

Figure 8. (a) Transit-timing variations, observed minus calculated for a constant ephemeris, $O-C$, and (b) both transit and eclipse timing variations (ETV), $O-C$, vs. transit/eclipse chronological order with estimated error bars. Note that panel (b) has a vertical scale that is 12 times larger than panel (a). The horizontal dotted lines are the average of the seven transits and seven eclipses; this is zero for the transits as we have subtracted off the best-fit transit ephemeris. The numbers above each data point show the number of periods before or after the zero point of our measured ephemeris; the points are not plotted as they occur in time, but are simply evenly spaced.

Table 1

Transit Parameters

\begin{tabular}{|c|c|c|c|c|c|c|c|c|c|c|}
\hline Phase & $\begin{array}{c}F_{*} \\
\text { (counts) }\end{array}$ & $\begin{array}{c}t_{\mathrm{c}} \\
\text { (BJD-2454000 days) }\end{array}$ & $\begin{array}{l}\sigma_{t_{\mathrm{c}}} \\
(\mathrm{s})\end{array}$ & $\begin{array}{l}\Delta t_{\mathrm{c}} \\
(\mathrm{s})\end{array}$ & $\begin{array}{c}b \\
\left(R_{*}\right)\end{array}$ & $\begin{array}{c}v \\
\left(R_{*} \text { day }^{-1}\right)\end{array}$ & $\begin{array}{c}\left(R_{p} / R_{*}\right)^{2} \\
(\%)\end{array}$ & $u_{1}$ & $\begin{array}{c}-a_{1},-a_{2} \\
\text { (counts) }\end{array}$ & $\begin{array}{c}\tau_{1}, \tau_{2} \\
\left(10^{-2} \text { days }\right)\end{array}$ \\
\hline 109.0 & 62רבק & $37.611919 \pm 0.000034$ & & & $.6694 \pm 0.0062$ & & & $0.08 \pm 0$ & & \\
\hline 1.0 & 6 & & 3 & -0 & & & & & & \\
\hline 2.0 & 6 & 00042 & 3.6 & & & & & & & \\
\hline 52.0 & 66758.8 & .000028 & 2.4 & & & & & & & \\
\hline 63.0 & 66995.3 & $9.207003 \pm 0.000036$ & 3. & 1. & $0.6594 \pm 0.0$ & $25.18 \pm$ & $2.4225 \pm 0.0$ & & & 0.532 \\
\hline 8.0 & 67385 & $29.971694 \pm 0$ & 2.9 & 1 & $641 \pm 0$ & 24.9 & $2.3984 \pm 0.0$ & $0.16=$ & & 0.4560 \\
\hline 159.0 & 67242.2 & $632.190128 \pm 0.000039$ & 3.4 & -10.4 & $0.6685 \pm 0.0060$ & $24.99 \pm 0.16$ & $2.3965 \pm 0.0074$ & $0.08 \pm 0.03$ & 715,669 & $0.4890,5.8697$ \\
\hline
\end{tabular}

Table 2

Eclipse Parameters

\begin{tabular}{lccccccc}
\hline \hline Phase & $\begin{array}{c}F_{*} \\
\text { (counts) }\end{array}$ & $\begin{array}{c}t_{\mathrm{c}} \\
\text { (BJD-2454000 days) }\end{array}$ & $\begin{array}{c}\sigma_{t_{\mathrm{c}}} \\
(\mathrm{s})\end{array}$ & $\begin{array}{c}\Delta t_{\mathrm{c}} \\
(\mathrm{s})\end{array}$ & $\begin{array}{c}F_{p} / F_{*} \\
(\%)\end{array}$ & $\begin{array}{c}-a_{1},-a_{2} \\
(\text { counts })\end{array}$ & $\begin{array}{c}\tau_{1}, \tau_{2} \\
\left(10^{-2} \text { days }\right)\end{array}$ \\
\hline-108.5 & 67466.9 & $38.722278 \pm 0.000265$ & 22.9 & 9.1 & $0.3345 \pm 0.0057$ & 0,0 & $0.0000,0.0000$ \\
0.5 & 66870.3 & $280.546423 \pm 0.000263$ & 22.7 & -32.5 & $0.3469 \pm 0.0060$ & 609,535 & $0.3793,4.5632$ \\
1.5 & 66808.5 & $282.765713 \pm 0.000350$ & 30.2 & 29.3 & $0.3420 \pm 0.0068$ & 255,118 & $0.1134,4.8741$ \\
51.5 & 66904.4 & $393.693798 \pm 0.000414$ & 35.8 & -26.2 & $0.3368 \pm 0.0042$ & 750,727 & $0.6350,6.1896$ \\
63.5 & 67097.2 & $420.317184 \pm 0.000287$ & 24.8 & 16.2 & $0.3623 \pm 0.0064$ & 759,647 & $0.5089,6.0830$ \\
157.5 & 67582.4 & $628.862649 \pm 0.000390$ & 33.7 & -30.8 & $0.3378 \pm 0.0091$ & 330,239 & $0.0310,5.6520$ \\
158.5 & 67318.3 & $631.081875 \pm 0.000313$ & 27.0 & 25.5 & $0.3477 \pm 0.0075$ & 737,685 & $0.5505,5.6994$ \\
\hline
\end{tabular}

scatter for the seven observations of $0.67 \%$, also consistent with being constant. We constrain the change in impact parameter to be $d b / d t=(-0.02 \pm 2.67) \times 10^{-5} R_{*}$ day $^{-1}$. Thus, our data indicate that the impact parameter and sky velocity of the transits remain constant to $<1 \%$ over a duration of 590 days.

\subsection{Transit and Eclipse Times}

We measured the transit and eclipse times for the seven transits and eclipses, shown in Figure 8, as well as in Tables 1 and 2. The errors on the transit times range from 2.4 to $3.6 \mathrm{~s}$ and are some of the most precise transit times ever measured, comparable to, or better than, the three HST transit times reported in Pont et al. (2007). We fit separate ephemerides to the transits and eclipses; the results are shown in Table 3. If we instead fit the transit times with the quadratic function: $t_{\mathrm{n}}=t_{0}+P n+\frac{1}{2} \dot{P} P n^{2}$, where $t_{\mathrm{n}}$ is the time of the $n$th
Table 3

Transit and Eclipse Ephemerides

\begin{tabular}{lcc}
\hline \hline & $T_{0}$ & $P$ \\
& $\left(\right.$ BJD $\left._{\text {UTC }}\right)$ & $($ days $)$ \\
\hline Transit & $2454279.436714 \pm 0.000015$ & $2.21857567 \pm 0.00000015$ \\
Eclipse & $2454279.437510 \pm 0.000125$ & $2.21857456 \pm 0.00000131$ \\
\hline
\end{tabular}

transit, and $\dot{P}$ is the change in period of the orbit, we find $\dot{P}=-0.06 \pm 0.02 \mathrm{~s} \mathrm{yr}^{-1}$. Since this is primarily due to the last data point, which may be an outlier, we do not view this as a significant detection.

The uncertainty on the transit times and eclipse times, as well as the derived ephemerides, is inversely proportional to depth of the transits and eclipses. This is due to the fact that the timing precision is proportional to the inverse of the flux gradient with 
time during ingress and egress. The ingress and egress durations are the same for the transit and eclipse (assuming a circular orbit), so the ratio of the flux gradient scales with the ratio of their depths. The ratio of the depths is proportional to the ratio of the surface brightness of the star to the surface brightness of the planet (limb darkening is weak for this star at $8 \mu \mathrm{m}$ ), so the transit time precisions are smaller than the eclipse precisions by the ratio of the surface brightness of the planet to the star, which is about $14.3 \%$, or a factor of 7.0.

Figure 8 shows the deviations from the ephemeris for both the transits and the eclipses. The transits have a scatter of $5.1 \mathrm{~s}$, which is very close the observational errors; there is no evidence in our data for TTVs over a period of 590 days. The eclipses also appear to be precisely periodic - their scatter with respect to the best-fit ephemeris is $27 \mathrm{~s}$, which is comparable to the errors on each data point. The period derived from the transits differs from that derived from the eclipses by only $0.1 \mathrm{~s}$ !

The eclipses appear $69 \pm 11 \mathrm{~s}$ later than $1 / 2$ of an orbital period after the transits. As discussed in Knutson et al. (2007), this is partly due to the light travel across the system, $30.8 \pm$ $0.6 \mathrm{~s}$ (this uncertainty is due to the uncertainty in stellar mass, $M_{*}=0.806 \pm 0.048 M_{\odot}$; Torres et al. 2008), while the remaining $38 \pm 11 \mathrm{~s}$ can be mostly accounted for by the hot spot on the planet causing an offset in the time of eclipse when the planet is modeled as a uniform disk, as shown in Section 6.5.

\subsection{Limits on the Presence of Companion Planets from Transit Timing}

These transit data show no significant timing variations, but from these we can constrain the maximum mass allowed of additional planets in the system. Transit timings are a particularly sensitive probe for planets in or near mean-motion resonance (MMR) and previous studies have ruled out Earth mass or super-Earth mass planets in low-order MMR for several systems. Prior TTV analyses of the HD189733 system (Hrudková et al. 2010; Miller-Ricci et al. 2008) used data with timing precision of order $30 \mathrm{~s}$ and were sensitive to planetary masses of near (and below) 1 Earth mass in favorable MMRs. Our Spitzer observations of HD189733 have nearly a factor of 10 better timing precision and consequently have improved sensitivity to secondary planets by that same factor. Here, we calculate the maximum mass that an additional planet could have based upon these transit data. To do so we note that the $\chi^{2}$ per degree of freedom of the timing residuals is slightly more than unity. We therefore scale the timing uncertainty by a factor of 1.5 and then multiply by two to achieve our $2 \sigma(\simeq 95 \%$ confidence level) upper bound on the timing variations.

Figure 9 shows 95\% confidence-level constraints on secondary planets with near circular orbits in this system based upon these data and the RV measurements from Boisse et al. (2009). These limits are derived from the analytic formula given in Agol et al. (2005) and Steffen \& Agol (2005). We do not attempt an in-depth numerical analysis of these transit times here-the robustness of limits derived from analytic formulae was demonstrated in Agol \& Steffen (2007), Nesvorný (2009), and Nesvorný \& Beaugé (2010). These data exclude planets above 2 Earth masses for any orbit that lies closer to the known planet than either the interior or exterior 2:1 MMR. The transittiming mass exclusion is superior than the exclusion from RV data for periods from 1 to 5 days, excluding all planets with masses greater than 3 Earth masses within this range. In addition they exclude planets with masses well below the mass of Mars-approximately 0.2 Mars masses or 2 Moon masses

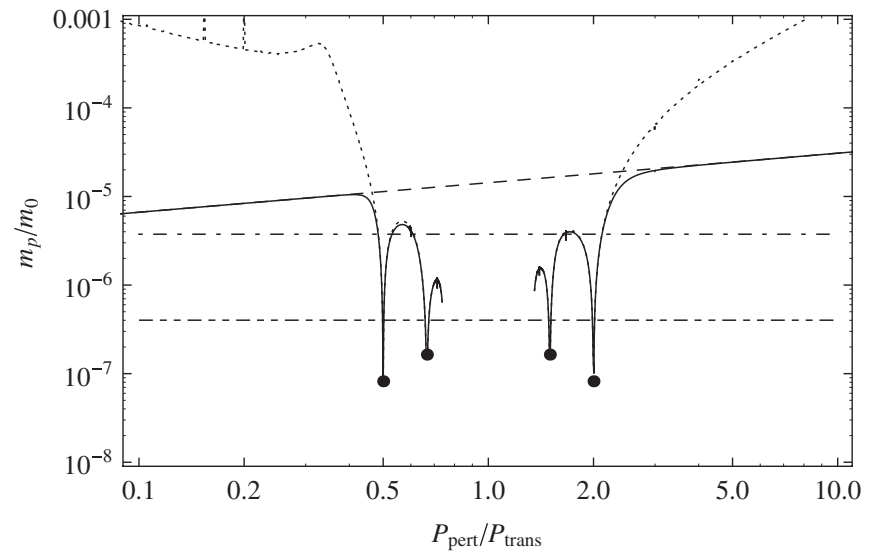

Figure 9. Constraints (95\% confidence level) on initially circular orbiting secondary planets in HD189733 as a function of the period ratio of the known planet based upon these transit data and the radial velocity measurements presented in Boisse et al. (2009). The dotted curve are the limits from a TTV analysis alone from Equations (A7) and (A8) in Agol et al. (2005). The dashed line is the expected sensitivity from $33 \mathrm{RV}$ measurements with $3.5 \mathrm{~m} \mathrm{~s}^{-1}$ precision calculated using Equation (2) from Steffen \& Agol (2005). The solid curve is the overall sensitivity from both RV and TTV measurements (summed in quadrature); the region above this curve is excluded. The solid dots are the variation in mean-motion resonance, $\approx P_{\text {trans }} m_{\mathrm{p}} / m_{\mathrm{t}}$, where $m_{\mathrm{t}, \mathrm{p}}$ are the masses of the transiting and perturbing planets (Agol et al. 2005). Finally, the horizontal dot-dashed and triple-dot-dashed lines correspond to the mass of the Earth and the mass of Mars, respectively.

at $95 \%$ percent confidence-in circularly orbiting $2: 1$ or $3: 2$ MMRs (interior or exterior). For non-circular orbits, the sensitivity generally increases. However, in low-order MMR the mass sensitivity can decline as much as a factor of 10 for eccentric orbits (see, for example, Agol \& Steffen 2007).

\subsection{Effect of Hot Spot on Secondary Eclipse Time}

As discussed in Knutson et al. (2007), the $8 \mu \mathrm{m}$ phase function indicates that the hottest point on the planet is offset from the sub-stellar point. This was predicted by Cooper \& Showman (2005), and is attributed to the advection of energy by a superrotating wind encircling the equator of the planet. This offset hot spot means that the ingress and egress of the secondary eclipse will have a shape that differs from our model, which utilizes a uniform disk. In particular, this means that the steepest portion of ingress and egress will be offset from the uniform disk case; since the hot spot is on the trailing side of the planet with respect to the direction of motion, this causes a delay in the eclipse time when fit with a uniform disk model (Charbonneau et al. 2005; Williams et al. 2006). In Knutson et al. (2007), we estimated that the hot spot would cause a delay of at most $20 \mathrm{~s}$; however, the fit to the phase function in that paper did not correct for stellar variation which caused the location of the hot spot to be underestimated, leading to an underestimated uniform time offset.

To estimate the magnitude of this effect, we used a simplified model of the longitudinal planet brightness which is discussed in Cowan \& Agol (2010b). Briefly, each position on the planet is treated as a parcel of gas which moves eastward, absorbing star light as it passes across the day side, all the while radiating with a time constant $\tau_{\text {rad }}$. This model can be parameterized by a single parameter, $\epsilon=\tau_{\text {rad }} / \tau_{\text {adv }}$, where $\tau_{\text {adv }}$ is the time it takes a parcel of gas to circle the planet. Small values of $\epsilon$ ("instant" reradiation) lead to darker night sides and dayside temperatures which are in equilibrium with the incident stellar flux. Large values of $\epsilon$ lead to nearly uniform temperatures at each latitude. 


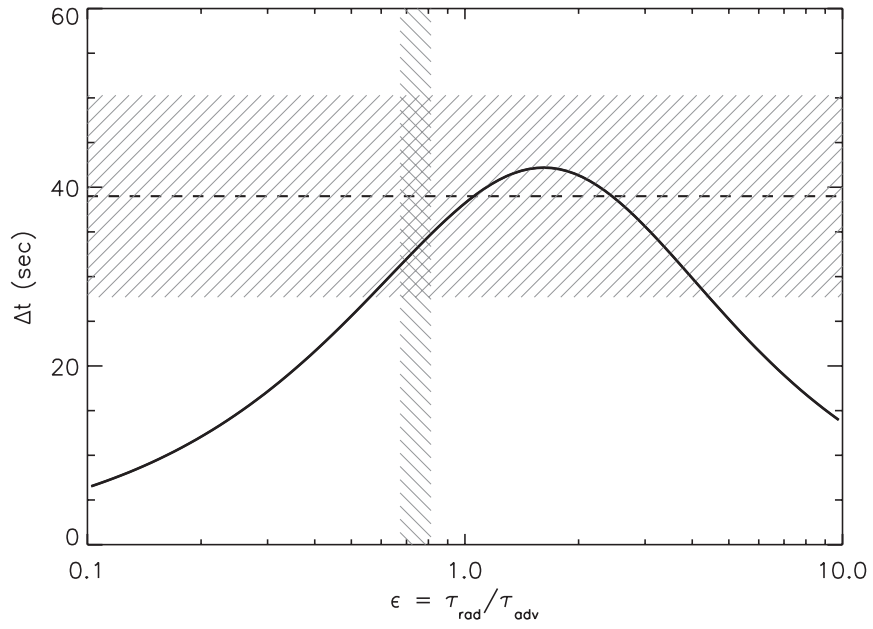

Figure 10. Timing offset for a hot-spot model as a function of the ratio of the radiative to advective timescales. The dashed line is the best-fit eclipse time offset after correction for light-travel time, and horizontal rectangular shaded region is the $1 \sigma$ confidence limit on this time. The vertical rectangular shaded region is the best-fit value of $\epsilon$ to the $8 \mu \mathrm{m}$ phase function, after correcting for stellar variability.

Thus, in the small or large $\epsilon$ limits we expect no timing offset since the day sides are symmetric. Only with $\epsilon \sim 1$ is there an offset hot spot causing a phase function which peaks before secondary eclipse, as well as a slight offset in the times of eclipse ingress and egress if fit with a uniform planet.

We computed the effect of $\epsilon$ on the time of secondary eclipse by solving for the planet dayside longitudinal surface brightness at the equator in the Rayleigh-Jeans limit and assuming a constant temperature with latitude. We computed the eclipse ingress and egress from this model for the planet surface brightness, we fit this simulated eclipse light curve with a model for the eclipse of a uniform planet, and from this best fit we determined the offset in the time of eclipse, the socalled uniform time offset defined by Williams et al. (2006). Figure 10 shows this time offset as a function $\epsilon$; a positive offset means that the secondary eclipse occurs later than expected for a uniform planet. The maximum offset predicted by this model is $43 \mathrm{~s}$, which agrees with the observed eclipse time offset. Our measured eclipse time is plotted as a dashed line in this figure, with the uncertainty indicated by the horizontal shaded rectangle.

The location of the peak in the planet phase function from Knutson et al. (2007) provides another constraint on the location of this hot spot, or equivalently on the value of $\epsilon$ (see Figure 11). We used the relation between the infrared and optical stellar variability derived in Section 5 to derive the change in stellar flux at $8 \mu \mathrm{m}$ during the phase-function measurement, about $0.6 \mathrm{mmag}$ over $26.6 \mathrm{hr}$. We then fit the last $2 / 3$ of the measured $8 \mu \mathrm{m}$ phase function to estimate $\epsilon$ (Figure 11); we discarded the first $1 / 3$ of the phase-function data since it is strongly affected by the ramp correction. We find a best-fit value of $\epsilon=0.74 \pm 0.07$, which we have also plotted as a vertical shaded region in Figure 10. This value of $\epsilon$ predicts a timing offset of $33 \mathrm{~s}$, which is consistent with the measured offset of $38 \pm 11$ s. Figure 12 shows a direct comparison of the secondary eclipse to the average of our seven secondary eclipses. The top panel shows the binned data as well as the best-fit secondary eclipse at $1 / 2$ orbital period after transit plus the $30.8 \mathrm{~s}$ lighttravel time delay (solid line), as well as the $\epsilon=0.74$ model with light-travel time delay (dashed line). The bottom panel shows

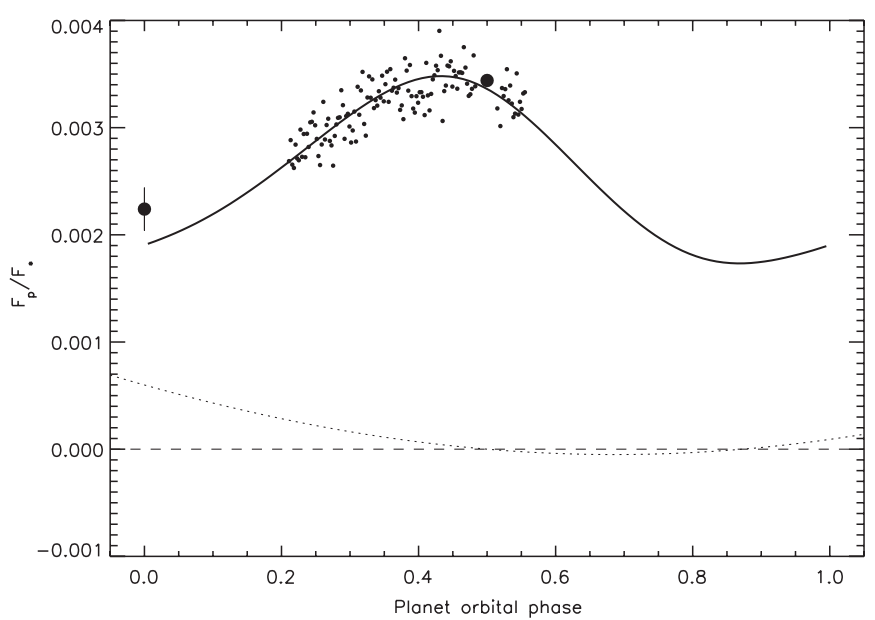

Figure 11. Planet phase function after correction for stellar variability vs. planet orbital phase. We only use the last $\sim 2 / 3$ of the phase function to avoid problems with the ramp correction, and we masked the secondary eclipse. The thick solid line is the best-fit model for planet variability with $\epsilon=0.74$. The dot with error bar on the left is our estimate of the nightside brightness from Equation (9). The dotted line shows our correction for stellar variability during the phase function.

the residuals binned into eleven bins: pre-ingress, post-egress, eclipse, and four bins each in ingress and egress; the residuals are plotted for the uniform planet model (diamonds) and $\epsilon=0.74$ model (filled circles with error bars). The uniform planet model shows points which are on average higher in ingress and lower in egress, which is a sign of the shifted hot spot. The hot-spot model provides a better fit to the data, although there is still scatter in the residuals which just reflects the low significance of the eclipse hot-spot detection (the uniform time offset is only $3.5 \sigma: 38 \pm 11 \mathrm{~s}$ ). Note that we have not optimized the hot-spot model, but only computed the light curve from the best fit to the phase function (which gives $\epsilon=0.74$ ).

Consequently, there is no evidence for non-zero $e \cos \omega$ in this system. For the estimated value of $\epsilon$, the remaining time offset is $6 \pm 11 \mathrm{~s}$, which yields $e \cos \omega=0.00005 \pm 0.00009$.

Another prediction of this model is the day-night brightness difference. For $\epsilon=0.74$, we predict a nightside brightness which is $57 \%$ of the dayside brightness (day defined at mideclipse; night at mid-transit). This corresponds to a decrease in brightness from the day to night side which is $0.15 \%$ of the stellar flux, which is very close to the value of $1.2 \pm$ 0.2 mmag derived in Section 5. The minimum planet brightness (for the visible hemisphere) divided by the maximum planet brightness for this best-fit model is $50 \%$, while the peak in observed planet brightness is $23^{\circ}, 0.065$ orbital periods, or $3.5 \mathrm{hr}$ before the secondary eclipse. On the planet, the hottest longitude is $13^{\circ}$ from the sub-stellar point; note that this differs from the hemispherically integrated peak due to asymmetry in the longitudinal dayside intensity. Figure 11 shows the phasefunction data after correction for stellar variation and binned by 1000 data points, versus planet orbital phase with the bestfit model for the planet variability, $\epsilon=0.74$, overplotted. Also plotted is our estimate of the nightside flux based on Equation (9).

\subsection{Transit Depth Variation}

In the fits to the transits, we allowed the depth of each transit to vary independently through the ratio of the planet to stellar radius, $p=R_{\mathrm{p}} / R_{*}$. This ratio also affects the duration of ingress and egress, but the amount of time spent in ingress 


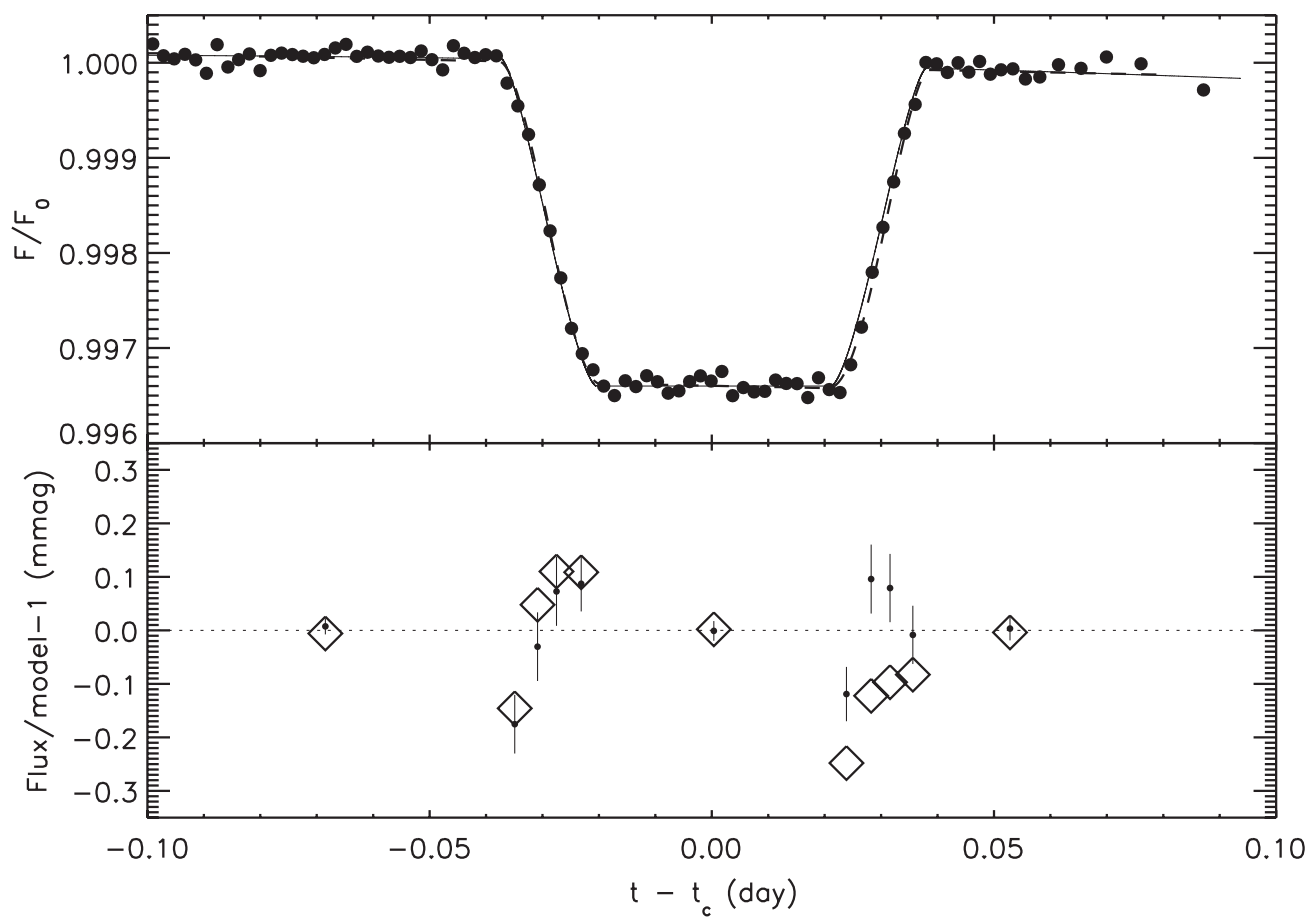

Figure 12. Plot of the average of seven eclipses (top panel) with best-fit uniform planet model, offset by $30.8 \mathrm{~s}$ after $1 / 2$ orbital period after the transit ephemeris (solid line), as well as the $\epsilon=0.74$ model with light-travel time delay (dashed line). The bottom panel shows the residuals binned into 11 bins: pre-ingress, post-egress, eclipse, and four bins each in ingress and egress; the residuals are plotted for the uniform planet model (diamonds) and $\epsilon=0.74$ model (filled circles with error bars).

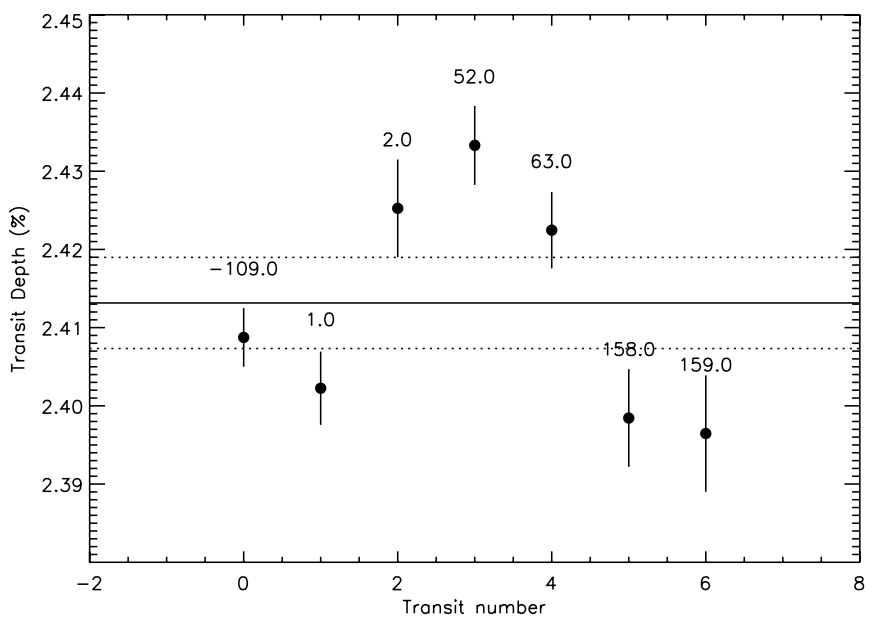

Figure 13. Transit depths measured for seven transits. The horizontal solid line measures the weighted mean transit depth, while the dotted lines give $1 \sigma$ uncertainty on this average value based on the scatter in the data. The numbers above each point indicate the orbital ephemeris.

or egress is small, so the primary effect is on the depth of transit. Figure 13 shows the derived transit depths, $p^{2}$, for the seven observed transits. There is evidence for variability in the transit depth-the uncertainty on the individual transit depths ranges from 37 to $74 \mu \mathrm{mag}$, while the scatter is $145 \mu \mathrm{mag}$. This corresponds to a scatter in the fractional variation of transit depth of $0.6 \%$, while the ratio of maximum to minimum is $1.5 \%$. Fitting the transit depths with a single value of $p$ gives a $\Delta \chi^{2}$ of 40.8 for 6 degrees of freedom. Thus, transit depth variation is detected with high significance.

We allowed the limb-darkening parameter to vary for each transit, which ranged from 0.08 to 0.13 for the seven transits, with an overall mean of $u_{1}=0.12 \pm 0.01$. Even though limb darkening is weaker in the infrared, an LTE Kurucz model atmosphere (Kurucz 1992) with parameters close to the values inferred for $\mathrm{HD} 189733, T_{\text {eff }}=5000 \mathrm{~K},[\mathrm{Fe} / \mathrm{H}]=0.0$, and $\log \left[g\left(\mathrm{~cm} \mathrm{~s}^{-2}\right)\right]=4.5$, predicts a linear limb-darkening coefficient of 0.136 , close to what we measure. We checked that the variation in transit depth is not due to variations in the best-fit limb-darkening parameter by holding the limbdarkening coefficient fixed at the mean value; this did not affect our measured values of transit depth.

In fact, the variation in transit depth is not necessarily due to a change in $p$. The average depth of each transit is given by $\delta=\left\langle I_{\mathrm{path}}\right\rangle \pi R_{\mathrm{p}}^{2} /\left(F_{*}+F_{\mathrm{p}}\right)$ (Mandel \& Agol 2002), where $\left\langle I_{\mathrm{path}}\right\rangle$ is the average surface brightness within the path of the planet across the star and $F_{\mathrm{p}}, F_{*}$ are the planet and stellar flux during transit. Although our model assumes a linear limb-darkening law, if the path of the planet passes over a region of the star with brighter than average surface brightness, then a larger depth will be inferred.

So, it is possible that the change in transit depth is due to changes in $\left\langle I_{\mathrm{path}}\right\rangle / F_{*}, R_{\mathrm{p}}, R_{*}$, or $F_{\mathrm{p}} / F_{*}$. Variations in $R_{\mathrm{p}}$ or $R_{*}$ seem unlikely to be responsible for the transit depth variation as this would require changes in radius of $0.3 \%$ for either body: either $220 \mathrm{~km}$ for the planet ( 1/3 of a thermal scale height) or $1600 \mathrm{~km}$ for the star. Both of these variations seem too large to be physically plausible. Variations in $\left\langle I_{\text {path }}\right\rangle / F_{*}$ due to fluctuations in $F_{*}$ are ruled out as the transit depth variations are uncorrelated with the optical stellar flux variations. Variations in $F_{\mathrm{p}}$ (the nightside planet flux) are less than 0.35 mmag relative to $F_{*}$, which is too small by a factor of 17 to be responsible for the transit depth variations. Thus, the most likely possibility is that the transit depth variations are due to a variation in the occulted stellar intensity, $\left\langle I_{\text {path }}\right\rangle$. This requires only a variation of $0.6 \%$ in the surface brightness of the path of the planet relative to the average stellar surface brightness, which is much smaller than the $12 \%$ change in surface brightness from center to limb inferred for the best-fit limb darkening. We have checked that 


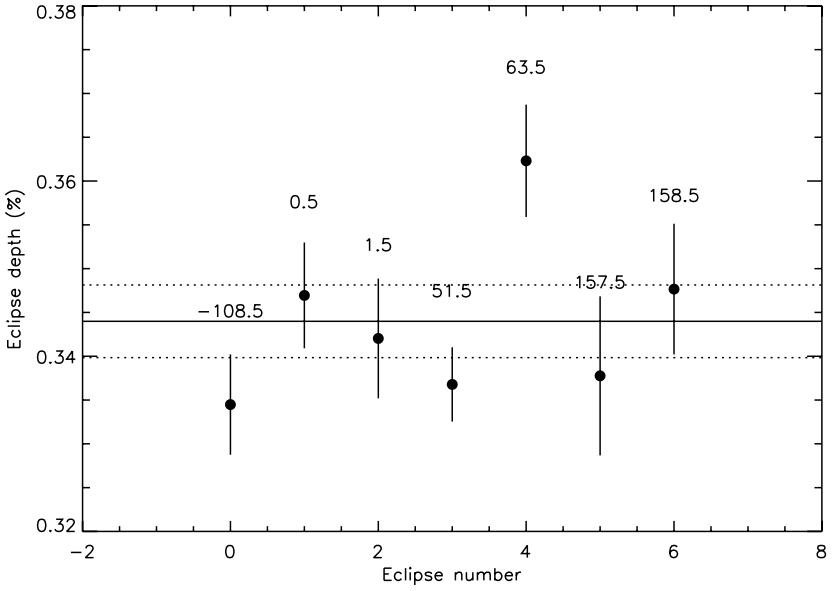

Figure 14. Eclipse depths measured for seven planet eclipses. The horizontal solid line measures the average transit depth, while the dotted lines give $1 \sigma$ uncertainty on this average value. The numbers above each point indicate the orbital ephemeris.

individual star spots are not responsible for this variation by computing the standard deviation of the residuals in transit divided by the square root of the counts, which is 1.153 , versus the same quantity computed out of transit, which is 1.154 , so there is no evidence for individual star spots causing this difference. Similar inferences have been drawn for Channels 1 and 3 by Beaulieu et al. (2008), while star spots are easier to detect in the optical where the contrast between star spots and the stellar disk is larger, and have already been detected for this star by Pont et al. (2007), who also resolved the shape and color dependence of the spots.

\subsection{Eclipse Depth Variation}

Figure 14 shows the measured eclipse depth in units of the stellar flux at mid-eclipse. The weighted mean eclipse depth is $0.344 \% \pm 0.0036 \%$ and the $\chi^{2}$ fit to the eclipse depths with this mean value is 14.6 for 6 degrees of freedom. The errors on each eclipse depth vary between $0.004 \%$ and $0.009 \%$, while the scatter in the depths is $0.01 \%$, so there is no detection of significant eclipse depth variability. This scatter corresponds to $2.7 \%$ variation of the mid-eclipse planet brightness; this can be taken as an upper limit on the planet variability at $68 \%$ confidence.

Although our eclipse depths do not exhibit significant variability, they only probe variability on timescales shorter than the baseline of the observations (roughly 2 years). To verify that our data do not show any time structure, we plot in Figure 15 the change in eclipse depth against the time between observations, for each pair of observations $(N \times(N-1) / 2=21$ for $N=7)$. We add uncertainties in quadrature to estimate the uncertainty on the flux differences. The resulting locus is flat, showing that measured eclipse depth is uncorrelated with the time of the observation. Note that this sets a limit not only astrophysical variability scenarios, but also systematic errors.

This limit on the variation in eclipse depths is sufficient to rule out the predicted variation computed for HD 189733b by Rauscher et al. (2008). The most extreme prediction they make is for their $\eta=0.05, \bar{U}=800 \mathrm{~m} \mathrm{~s}^{-1}$ model which has a standard deviation of $\sim 8 \%$ in the dayside brightness, with the largest excursions of $20 \%$. The largest difference in brightness we see is $8 \%$, while the scatter in planet brightness is $2.7 \%$, so by both measures the observed variation is a factor of $\sim 3$ smaller than the predictions of this particular model.

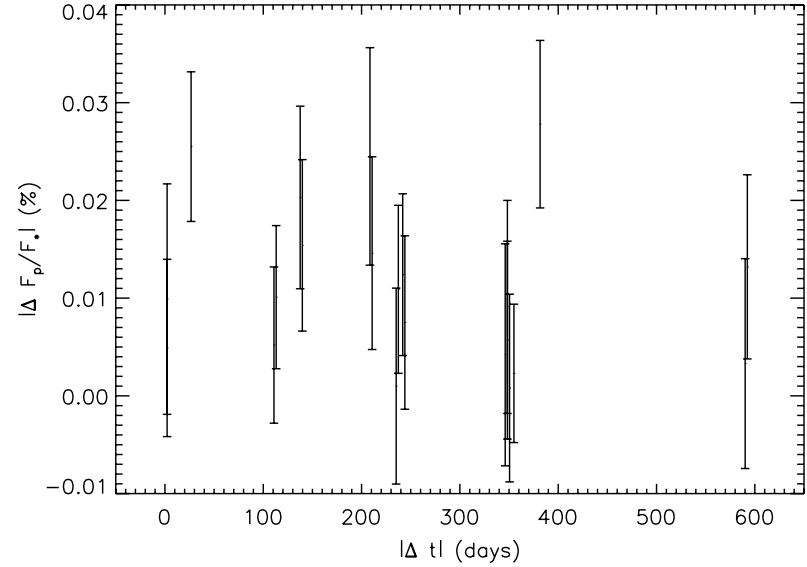

Figure 15. For each pair of eclipse observations, we show here the change in eclipse depth as a percent, vs. the time between the observations. If the eclipse depths showed time correlation-due to either astrophysical variability on some characteristic timescale or detector systematics - this plot would show a rise. The flat distribution is consistent with Gaussian variations at the level of a few percent.

Other models predict smaller variations in the dayside brightness, such as Showman et al. (2009b) who compute the $8 \mu \mathrm{m}$ brightness variation for HD 189733 b should be less than $1 \%$. Our upper limits are consistent with this model, but unfortunately do not constrain the model due to our uncertainties that are larger than the predicted variations.

With upper limits on both day and nightside variability, it is worth asking which of these puts stronger constraints on the planet's physical properties. Consider the simple model of Cowan \& Agol (2010a), which parameterizes the planet's day and nightside brightnesses in terms of the planet's Bond albedo and recirculation efficiency. One can treat brightness variability — of the day or night — as being due to changes in albedo and/or changes in recirculation efficiency, and compute how these affect the day and nightside brightness. We find that the dayside variability upper limit provides a better constraint on variation in the Bond albedo or recirculation efficiency than does the nightside variability limit, which is $\sim 6 \times$ larger than the dayside limit.

Another possible origin of planet dayside variability are transient local variations in the surface brightness, for example, due to large-scale "storms." We use a toy model where the planet's day side has a uniform temperature, $T_{\mathrm{d}}$, except for a storm with covering fraction $0<f<1$ (the $y$-axis) and temperature difference $\Delta T$ from mean dayside temperature (the $x$-axis). Figure 16 shows exclusion limits on the largest putative storm that could form or dissipate without appearing in our data. The increasingly dark shades of gray denote areas of parameter space excluded at $1 \sigma$ through $12 \sigma$. According to Showman et al. (2009a), the radius of deformation for HD 189733 b is 0.3 of the planetary radius, an order of magnitude larger than for Jupiter. The covering fraction for a typical storm on such a planet would be $f=0.1$, for which we cannot rule out storms differing by $324 \mathrm{~K}$ (68\% confidence) from the average dayside temperature. For comparison, Jupiter's Great Red Spot has a filling fraction of roughly 0.03 , and a temperature $12 \mathrm{~K}$ cooler than the rest of the planet. The bottom line is that our data rule out only the most extreme weather fluctuations on HD 189733b.

\subsection{System Parameters}

Due to the high precision of our data and weak limb darkening in the infrared, we can considerably improve the determination 


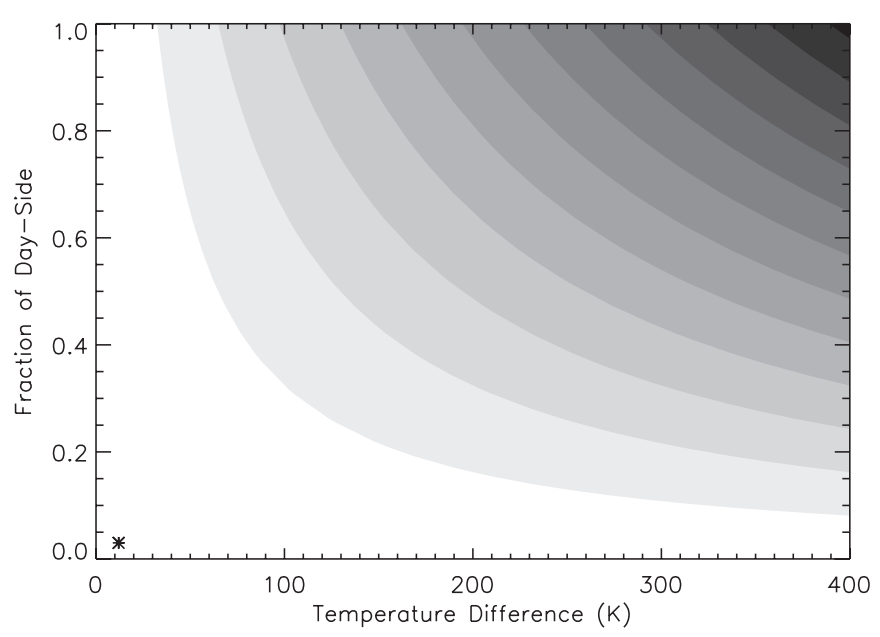

Figure 16. Exclusion plot for the covering fraction and temperature contrast of a putative storm on the day side of HD 189733b. The top right corner of the plot is excluded at $12 \sigma$. For comparison, Jupiter's Great Red Spot has a filling fraction of roughly 0.03 , and a temperature $12 \mathrm{~K}$ cooler than the rest of the planet (asterisk).

of certain stellar and planet parameters for this system from our data. Since both the small inferred value of $e \cos \omega$ and theoretical predictions indicate that $e$ should be close to zero, we set $e=0$ in deriving the system parameters. The uncertainties on the stellar and planet parameters are computed for each transit or eclipse by computing the system parameters from the model parameters from each simulation (using the relations in Winn 2010), computing the standard deviation of the results from the simulations as an estimate of the errors on each parameter, and then taking a weighted mean of all transits/eclipses to obtain the final mean value of the best-fit parameters.

Table 4 presents the system parameters determined from all 14 transits and eclipses. We have focused on parameters that are most directly constrained from the photometry, which are either dimensionless, or have units of density. Compared to the values derived in Torres et al. (2008) and Pont et al. (2007), the uncertainties on our values are smaller by a factor of $2-10$. For the planet surface gravity, $g_{\mathrm{p}}$, we use the velocity semi-amplitude $K=200.56 \pm 0.88 \mathrm{~m} \mathrm{~s}^{-1}$, derived by Boisse et al. (2009), and for the planet density, $\rho_{\mathrm{p}}$, we use the stellar mass $M_{*}=0.806 \pm 0.048 M_{\odot}$ given in Torres et al. (2008), propagating the uncertainties assuming they are uncorrelated and Gaussian.

\section{DISCUSSION AND CONCLUSIONS}

The analysis of 14 transits and eclipses in this paper has made several improvements to the data reduction and modeling; in particular, we have found a better function for fitting the detector ramp of IRAC Channel 4, a double exponential. The scatter in the residuals is approaching that of photon counting errors, similar to the precision achieved in other IRAC observations (e.g., Todorov et al. 2010), but for a brighter source star, and the residuals show very little evidence of red noise. These technical developments have allowed us to make a better correction for stellar variability, and have given us better constraints on the parameters of this system.

As HD 189733 is the first planet system in which the phase variation has been measured at high significance, it provides some of the tightest constraints on the atmospheric dynamics of an extrasolar planet. Our observations of an additional six transits and eclipses presented here allow us to place additional
Table 4

Best-fit System Parameters

\begin{tabular}{lcc}
\hline \hline Parameter & Best Fit & Units \\
\hline$a / R_{*}$ & $8.863 \pm 0.020$ & \\
$b / R_{*}$ & $0.6631 \pm 0.0023$ & \\
$i$ & $85.710 \pm 0.024$ & $\mathrm{deg}$ \\
$e \cos \omega$ & $0.000050 \pm 0.000094$ & \\
$u_{1}$ & $0.118 \pm 0.010$ & \\
$\rho_{*}$ & $2.670 \pm 0.017$ & $\mathrm{~g} \mathrm{~cm}^{-3}$ \\
$R_{p} / R_{*}$ & $0.155313 \pm 0.000188$ & \\
$F_{p} / F_{*}$ & $0.3440 \pm 0.0036$ & $\%$ \\
$g_{p}$ & $2145.9 \pm 13.5$ & $\mathrm{~cm} \mathrm{~s}^{-2}$ \\
$\rho_{p}$ & $0.943 \pm 0.024$ & $\mathrm{~g} \mathrm{~cm}^{-3}$ \\
\hline
\end{tabular}

constraints on the longitudinal brightness distribution of the planet at $8 \mu \mathrm{m}$. In particular, we have improved the measurement of the correlation between the optical, $(b+y) / 2$ band, and $8 \mu \mathrm{m}$ variations in the star over the correlation measured in Knutson et al. (2009), giving a correlation that agrees better with predictions of star spot models. This measured correlation allows us to derive a better correction for the stellar variation during the observation of Knutson et al. (2007), giving us a better measurement of the planet's phase function. In particular, we find that the peak planet flux at $8 \mu \mathrm{m}$ occurs $3.5 \mathrm{hr}$ before secondary eclipse, which is $1.2 \mathrm{hr}$ before the value derived in Knutson et al. (2007) without correction for stellar variation; this is consistent within the errors given in that paper, which were dominated by the ramp correction. This measured phase function predicts a $33 \mathrm{~s}$ delay of the secondary eclipse when fit with a uniform planet model, which is consistent with the $38 \pm 11 \mathrm{~s}$ delay that has been measured after correcting for light-travel time across the system. This confirms that the phase variation is indeed due to the planet, and gives a crude eclipse mapping of the planet detected the $3.5 \sigma$ level, as first pointed out by Williams et al. (2006). It is significant that-for the same high-quality photometry-phase-function mapping (Cowan \& Agol 2008) is more effective at locating the planet's primary hot spot than eclipse mapping (Williams et al. 2006). This is because the duration of eclipse ingress or egress is shorter by a factor $\sim R_{\mathrm{p}} /(2 \pi a)$ than the planet's orbital period, while the changes in brightness used by both techniques are comparable. The superior leverage of phase-function mapping will become even more marked as interest shifts toward smaller planets in longer orbits. That said, the two mapping techniques suffer from different degeneracies and different impacts of systematic errors and stellar variability, so when possible one will want to use both. We also confirm the phase variation by measuring the difference between the fluxes at transit and eclipse, and we find the night side is fainter by $1.2 \pm 0.2 \mathrm{mmag}$, or about $64 \%$ of the brightness of the day side. All of these constraints are consistent with a model in which the gas circulating the planet has a radiative cooling timescale which is comparable to the advection timescale; we find $\tau_{\text {rad }} / \tau_{\text {adv }} \sim 0.74$ by fitting the phase function.

The larger offset in the time of peak planet flux is also in better agreement with the predictions of Showman et al. (2009b) who found that to obtain agreement with the smaller offset of Knutson et al. (2007) they required an inner boundary of their atmosphere that was rotating more slowly than synchronous rotation; instead of a sub-synchronous core, this may be indicative of slower wind speeds due to magnetic drag near the $8 \mu \mathrm{m}$ photosphere (Perna et al. 2010). The sub-synchronously rotating and $5 \times$ solar 
abundance models of Showman et al. (2009b) predict a peak brightness at $8 \mu \mathrm{m}$ which is $20^{\circ}-30^{\circ}$ before secondary eclipse, which agrees well with our new estimate of $27^{\circ}$. The same models also predict a dayside brightness (mid-transit) which is $0.33 \%-0.35 \%$ of the star's brightness, consistent with our measured value of $0.344 \% \pm 0.004 \%$. The nightside brightness at $8 \mu \mathrm{m}$ predicted by the models is $0.17 \%-0.18 \%$ of the stellar brightness, which is consistent with our measured value of $0.22 \% \pm 0.05 \%$. Their models also predict very small variations in the secondary eclipse depth of less than $1 \%$, which is consistent with our upper limit of $2.7 \%$. The lack of variation of the atmosphere indicates that the assumptions used in creating longitudinal maps of planets from phase functions are likely valid (Cowan \& Agol 2008).

The time delay for the secondary eclipse can be completely accounted for by the light-travel time of the system and delay of ingress and egress due to a hot spot on the planet which is offset longitudinally. Consequently, there is no evidence for a non-zero $e \cos \omega$, and we can place a limit of $e \cos \omega=$ $0.00005 \pm 0.00009$. If the orbit of this planet is nearly circular, which the small value of $e \cos \omega$ would indicate, then the interior is likely also synchronously rotating, which seems to agree with the Showman et al. (2009b) predictions for the phase function.

We have detected $8 \mu \mathrm{m}$ limb darkening of the star at high significance, $\sim 10 \sigma$, which agrees with predictions of stellar atmosphere models. However, the individual transits vary in depth, which we hypothesize may be due to variation of the stellar surface brightness that is occulted by the planet. This is not surprising given the strong optical variations of this star which indicate a significant presence of star spots. This variation needs to be accounted for in creating spectral absorption profiles of transiting planets. If the data taken are non-simultaneous, the variation in stellar surface brightness could affect the inferred depth of transit differently at different wavelengths, leading to systematic errors in comparison to model predictions; even simultaneous data might be affected by the star spot color. This is a stronger effect at shorter wavelengths; for example, the contrast in surface brightness of $4000 \mathrm{~K}$ star spots in the IRAC Channel $1(3.6 \mu \mathrm{m})$ and Channel $2(4.5 \mu \mathrm{m})$ should be 20\%-40\% higher than for Channel 4 for this star; thus fluctuations in transit depth could approach $2 \%$ in these bands. In addition, this limits the possibility of constraining the variations in transit depth due to planet oblateness (Carter \& Winn 2010).

Due to our highly precise transit times spaced over a wide range in time, the ephemeris we derive is one of the most precise for any transiting planet. The high precision is due to the weak limb-darkening, stable instrument (thanks to the Earth-trailing orbit of Spitzer which leads to stable thermal properties and no occultation of targets by the Earth, as occurs with the $H S T$ ), allowing a $3 \mathrm{~s}$ precision for transit times. Our ephemeris has a precision that is $>10$ times better for the period than that reported in Pont et al. (2007), and agrees with their reported period within $\sim 2.8 \sigma$ : their period is longer by $0.46 \pm 0.17 \mathrm{~s}$. Our ephemeris predicts times of transit that are $-3.3 \pm 5.0,3.5 \pm 5.0$, and $12.6 \pm 3.5 \mathrm{~s}$ after their three transit times in Pont et al. (2007). We detect no strong evidence for TTVs in our data, and we estimated from analytic formulae the upper mass limits on the presence of companion planets in this system, improving upon the limits placed by Miller-Ricci et al. (2008) and Hrudková et al. (2010) by a factor of $\sim 10$. Theories of the evolution of short-period planets due to tidal effects and interaction with turbulence in the protoplanetary disk indicate that they should evolve out of MMR, so the lack of detected TTVs may not be surprising, especially for interior perturbing planets (Fabrycky 2009; Adams et al. 2008; Terquem \& Papaloizou 2007; Papaloizou \& Terquem 2010).

In summary, the excellent stability of the Spitzer Space Telescope, and in particular Channel 4 of the IRAC camera, has enabled near photon-limited photometric errors, and submillimagnitude variations over a period 1.6 years. This has enabled a better calibration of the contribution of stellar variability at $8 \mu \mathrm{m}$, which has allowed us to measure the nightside planet brightness, and has shifted our estimate of the peak of the phase function.

This work is based in part on observations made with the Spitzer Space Telescope, which is operated by the Jet Propulsion Laboratory (JPL), California Institute of Technology under contract with NASA. Support for this work was provided by NASA through an award issued by JPL/Caltech. E.A. acknowledges the hospitality of the Institute for Theory and Computation at the Harvard-Smithsonian Center for Astrophysics, the Michigan Center for Theoretical Physics, and the Kavli Institute for Theoretical Physics where portions of this work were completed. H.A.K. is supported by a fellowship from the Miller Institute for Basic Research in Science. This research was supported in part by the National Science Foundation under grant No. NSF PHY05-51164 and CAREER grant No. 0645416.

\section{REFERENCES}

Adams, F. C., Laughlin, G., \& Bloch, A. M. 2008, ApJ, 683, 1117

Agol, E., Cowan, N. B., Bushong, J., Knutson, H., Charbonneau, D., Deming, D., \& Steffen, J. H. 2009, in IAU Symp. 253, Transiting Planets, ed. F. Pont,

D. Sasselov, \& M. Holman (Cambridge: Cambridge Univ. Press), 209

Agol, E., \& Steffen, J. H. 2007, MNRAS, 374, 941

Agol, E., Steffen, J., Sari, R., \& Clarkson, W. 2005, MNRAS, 359, 567

Baines, E. K., van Belle, G. T., ten Brummelaar, T. A., McAlister, H. A., Swain,

M., Turner, N. H., Sturmann, L., \& Sturmann, J. 2007, ApJ, 661, L195

Bakos, G. Á., Pál, A, Latham, D. W., Noyes, R. W., \& Stefanik, R. P. 2006a, ApJ, 641, L57

Bakos, G. Á., et al. 2006b, ApJ, 650, 1160

Barman, T. S. 2008, ApJ, 676, L61

Barnes, J. R., Barman, T. S., Prato, L., Segransan, D., Jones, H. R. A., Leigh, C. J., Collier Cameron, A., \& Pinfield, D. J. 2007, MNRAS, 382, 473

Beaulieu, J. P., Carey, S., Ribas, I., \& Tinetti, G. 2008, ApJ, 677, 1343

Boisse, I., et al. 2009, A\&A, 495, 959

Bouchy, F., et al. 2005, A\&A, 444, L15

Burrows, A., Budaj, J., \& Hubeny, I. 2008, ApJ, 678, 1436

Carter, J. A., \& Winn, J. N. 2010, ApJ, 709, 1219

Charbonneau, D., Knutson, H. A., Barman, T., Allen, L. E., Mayor, M., Megeath, S. T., Queloz, D., \& Udry, S. 2008, ApJ, 686, 1341

Charbonneau, D., et al. 2005, ApJ, 626, 523

Cooper, C. S., \& Showman, A. P. 2005, ApJ, 629, L45

Cowan, N. B., \& Agol, E. 2008, ApJ, 678, L129

Cowan, N. B., \& Agol, E. 2010a, arXiv:1001.0012

Cowan, N. B., \& Agol, E. 2010b, ApJ, submitted

Cowan, N. B., Agol, E., \& Charbonneau, D. 2007, MNRAS, 379, 641

Deming, D. 2009, in IAU Symp. 253, Transiting Planets, ed. F. Pont, D. Sasselov, \& M. Holman (Cambridge: Cambridge Univ. Press), 197

Deming, D., Harrington, J., Seager, S., \& Richardson, L. J. 2006, ApJ, 644, 560

Désert, J., Lecavelier des Etangs, A., Hébrard, G., Sing, D. K., Ehrenreich, D., Ferlet, R., \& Vidal-Madjar, A. 2009, ApJ, 699, 478

Eastman, J., Siverd, R., \& Gaudi, B. S. 2010, arXiv:1005.4415

Fabrycky, D. C. 2009, in IAU Symp. 253, Transiting Planets, ed. F. Pont, D. Sasselov, \& M. Holman (Cambridge: Cambridge Univ. Press), 173

Fazio, G. G., et al. 2004, ApJS, 154, 10

Grillmair, C. J., Charbonneau, D., Burrows, A., Armus, L., Stauffer, J., Meadows, V., Van Cleve, J., \& Levine, D. 2007, ApJ, 658, L115

Harrington, J., Luszcz, S., Seager, S., Deming, D., \& Richardson, L. J. 2007, Nature, 447, 691

Henry, G. W., \& Winn, J. N. 2008, AJ, 135, 68

Holman, M. J., \& Murray, N. W. 2005, Science, 307, 1288

Hrudková, M., et al. 2010, MNRAS, 403, 2111 
Kipping, D. M., \& Tinetti, G. 2010, MNRAS, in press

Knutson, H. A., et al. 2007, Nature, 447, 183

Knutson, H. A., et al. 2009, ApJ, 690, 822

Kurucz, R. L. 1992, in IAU Symp. 149, The Stellar Populations of Galaxies, ed. B. Barbuy \& A. Renzini (Cambridge: Cambridge Univ. Press), 225

Laughlin, G., Deming, D., Langton, J., Kasen, D., Vogt, S., Butler, P., Rivera, E., \& Meschiari, S. 2009, Nature, 457, 562

Lecavelier Des Etangs, A., Pont, F., Vidal-Madjar, A., \& Sing, D. 2008, A\&A, 481, L83

Madhusudhan, N., \& Seager, S. 2009, ApJ, 707, 24

Mandel, K., \& Agol, E. 2002, ApJ, 580, L171

Markwardt, C. B. 2009, in ASP Conf. Ser. 411, Astronomical Data Analysis Software and Systems XVIII, ed. D. A. Bohlender, D. Durand, \& P. Dowler (San Francisco, CA: ASP), 251

Miller-Ricci, E., et al. 2008, ApJ, 682, 593

Moutou, C., et al. 2007, A\&A, 473, 651

Nesvorný, D. 2009, ApJ, 701, 1116

Nesvorný, D., \& Beaugé, C. 2010, ApJ, 709, L44

Papaloizou, J. C. B., \& Terquem, C. 2010, MNRAS, 405, 573

Perna, R., Menou, K., \& Rauscher, E. 2010, ApJ, 719, 1421

Pont, F., Knutson, H., Gilliland, R. L., Moutou, C., \& Charbonneau, D. 2008, MNRAS, 385, 109

Pont, F., et al. 2007, A\&A, 476, 1347

Rauscher, E., \& Menou, K. 2010, ApJ, 714, 1334

Rauscher, E., Menou, K., Cho, J., Seager, S., \& Hansen, B. M. S. 2007, ApJ, 662, L115
Rauscher, E., Menou, K., Cho, J., Seager, S., \& Hansen, B. M. S. 2008, ApJ, 681, 1646

Redfield, S., Endl, M., Cochran, W. D., \& Koesterke, L. 2008, ApJ, 673, L87

Showman, A. P., Cho, J., \& Menou, K. 2009a, arXiv:0911.3170

Showman, A. P., Fortney, J. J., Lian, Y., Marley, M. S., Freedman, R. S., Knutson, H. A., \& Charbonneau, D. 2009b, ApJ, 699, 564

Showman, A. P., \& Guillot, T. 2002, A\&A, 385, 166

Sing, D. K., Désert, J., Lecavelier Des Etangs, A., Ballester, G. E., Vidal-Madjar, A., Parmentier, V., Hebrard, G., \& Henry, G. W. 2009, A\&A, 505, 891

Steffen, J. H., \& Agol, E. 2005, MNRAS, 364, L96

Swain, M. R., Vasisht, G., \& Tinetti, G. 2008, Nature, 452, 329

Swain, M. R., Vasisht, G., Tinetti, G., Bouwman, J., Chen, P., Yung, Y., Deming, D., \& Deroo, P. 2009, ApJ, 690, L114

Terquem, C., \& Papaloizou, J. C. B. 2007, ApJ, 654, 1110

Tinetti, G., et al. 2007, Nature, 448, 169

Todorov, K., Deming, D., Harrington, J., Stevenson, K. B., Bowman, W. C., Nymeyer, S., Fortney, J. J., \& Bakos, G. A. 2010, ApJ, 708, 498

Torres, G., Winn, J. N., \& Holman, M. J. 2008, ApJ, 677, 1324

Triaud, A. H. M. J., et al. 2009, A\&A, 506, 377

Werner, M. W., et al. 2004, ApJS, 154, 1

Williams, P. K. G., Charbonneau, D., Cooper, C. S., Showman, A. P., \& Fortney, J. J. 2006, ApJ, 649, 1020

Winn, J. N. 2010, arXiv:1001.2010

Winn, J. N., et al. 2006, ApJ, 653, L69

Winn, J. N., et al. 2007, AJ, 133, 1828 